\title{
Flux-corrected transport for full-waveform inversion
}

\author{
Mahesh Kalita and Tariq Alkhalifah \\ Department of Physical Science and Engineering, King Abdullah University of Science and Technology, Thuwal 23955-6900, Saudi Arabia. \\ E-mail:mahesh.kalita.2@kaust.edu.sa
}

Accepted 2019 February 22. Received 2019 February 18; in original form 2018 May 8

\begin{abstract}
SUMMAR Y
Conventional full-waveform inversion (FWI) often fails to retrieve the unknown model parameters from noisy seismic data. A successful FWI implementation usually requires to follow a multistage recovery approach, starting from the retrieval of the lower model wavenumbers (tomography) to those with the higher resolutions (migration). Here, we propose a new method based on the flux-corrected transport (FCT) technique often used in computational fluid dynamics for the removal of instabilities in a shock profile. FCT involves three finite-difference steps: a transport, a diffusion and an antidiffusion process. This third step involves non-linear operators such as maximum and minimum, which are non-differentiable in a classic sense. However, since the seismic source wavelet and the corresponding wavefield are relatively smooth and continuous in nature without any strong ripples like shock waves, we exclude the non-linear step from FCT, which allows us to evaluate the novel FWI gradient efficiently. As a result, we achieve a converging FWI model by gradually reducing the diffusive fluxcorrection. We demonstrate the versatility of FCT-based FWI on a noisy synthetic data set from the Marmousi II model and a marine field data set from offshore Australia.
\end{abstract}

Key words: numerical flux; finite difference methods; acoustic wave equation; full-waveform inversion; flux-corrected transport.

\section{INTRODUCTION}

In recent years, research on full-waveform inversion (FWI) has generated significant amounts of interest because of its potential to recover fine-scale heterogeneities of the Earth's interior from seismic data (Lailly 1983; Tarantola 1984; Pratt 1990). However, the Earth's complexity and the non-linear nature of scattered wavefields lay down theoretical and computational challenges when using FWI to obtain a subsurface model without any artifacts (Virieux \& Operto 2009). These artefacts are mostly due to the cycle-skipping problem of FWI (predicted and observed seismic recordings differ by more than half a cycle), and they lead the inversion to produce a local, rather than the global minimum. ${ }^{1}$ As a consequence, FWI can recover a model that may be worse than the starting model. Therefore, FWI gets deprived of its ultimate goal, that is: to retrieve fine details of the subsurface properties from seismic data, irrespectively of any degree of incorrectness in the initial models of consideration.

Over the last two decades, numerous techniques have been proposed to alleviate the dependence of FWI on the selection of initial models. The most widely used strategy is a hierarchical scaling of

\footnotetext{
${ }^{1}$ On the framework of local non-linear optimization problem, FWI honors single-scattering approximation which is known as Born approximation, and, hence cannot render non-linear model perturbations from the seismic data. As a result, FWI suffers from endemic cycle skipping problem.
}

the available frequencies in the data set (Bunks et al. 1995; Sirgue \& Pratt 2004; Boonyasiriwat et al. 2009; Yuan \& Simons 2014). This strategy involves gradually appending the high frequency details of the data to the inversion results of the low frequency. Unfortunately, this inefficient strategy often fails in a complicated field data set. Fortunately, strategies that integrate image-domain inversion such as migration velocity analysis to data domain schemes (i.e. FWI), expedite the prospect of converging to the global minimum (Symes 2008; Biondi \& Almomin 2014; Berkhout 2014; Allemand \& Lambaré 2015; Alkhalifah \& Wu 2016) with a trade off in computational cost and various implementation difficulties including separation of model scales.

In general, FWI minimizes an objective function with multiple local minima. Therefore, FWI, more often than not, falls into one of the many local minima, depending on the starting model. As a result, people look for objective functions that can keep convexity for large model perturbations, as an alternate to the conventional $L^{2}$ norm of the difference of observed and synthetic data. As a result, a wide range of objective functions such as envelope-based (Bozdağ et al. 2011; Wu et al. 2014; Oh \& Alkhalifah 2018), deconvolution-based (Luo et al. 2011; Warner \& Guasch 2016; Choi \& Alkhalifah 2018), optimal transport-based (Engquist \& Froese 2013; Métivier et al. 2016; Yang et al. 2018), auxiliary bump functional (Bharadwaj et al. 2016), weighted correlation-based (Van Leeuwen \& Mulder 2010; Wu \& Alkhalifah 2017) and so on attempt to deliver a converging model in conjunction with FWI. To expedite the convergence, 


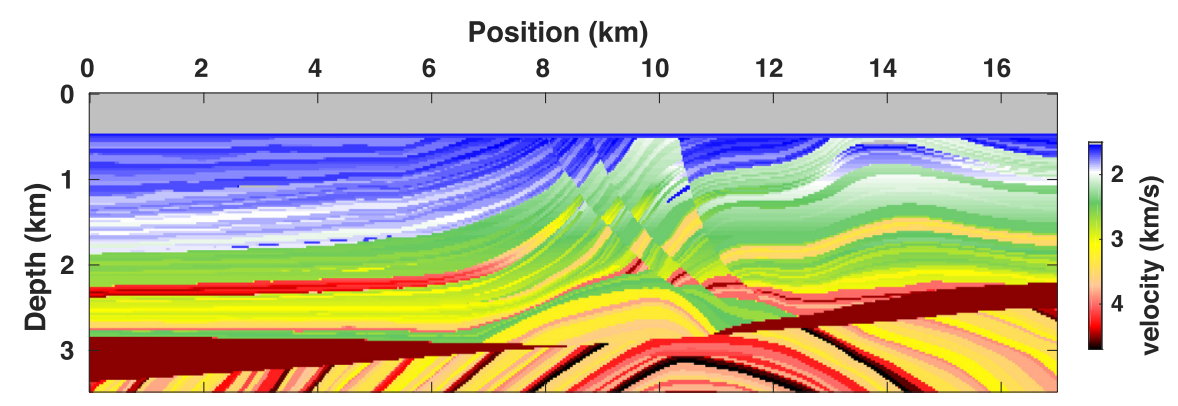

Figure 1. Marmousi II model.
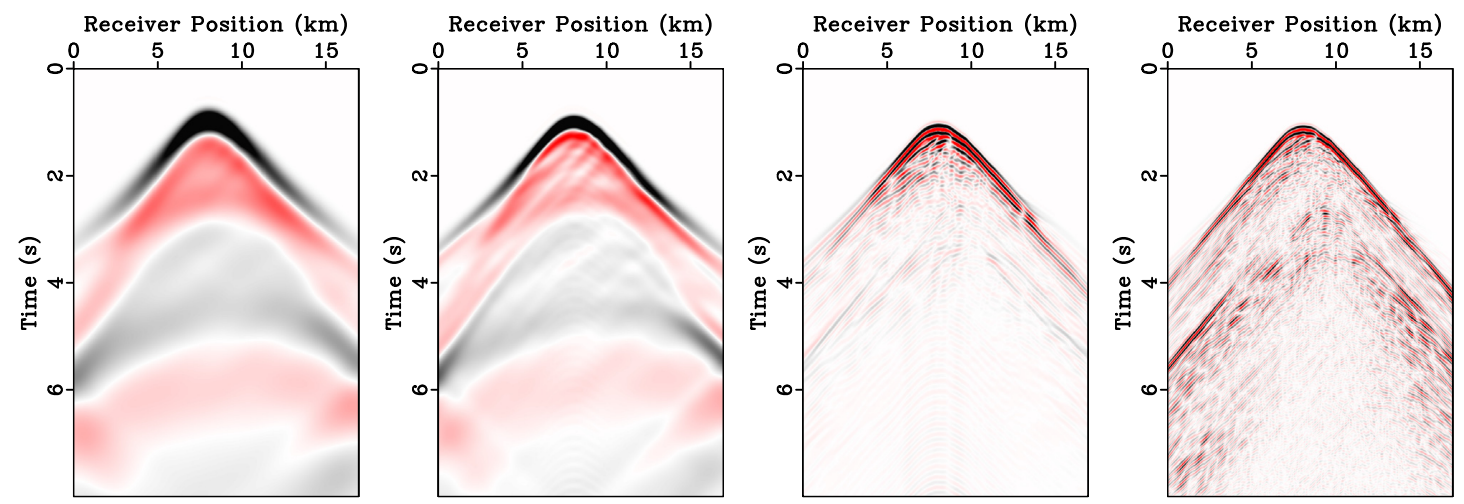

Figure 2. Wavefield recorded at $z=2.0 \mathrm{~km}$ depth with the source at $x=8.0 \mathrm{~km}$ for the model in Fig. 1. From left to right: $\xi=\{0.15,0.05,0.005,0\} . \xi=0$ suggests conventional forward modelling.

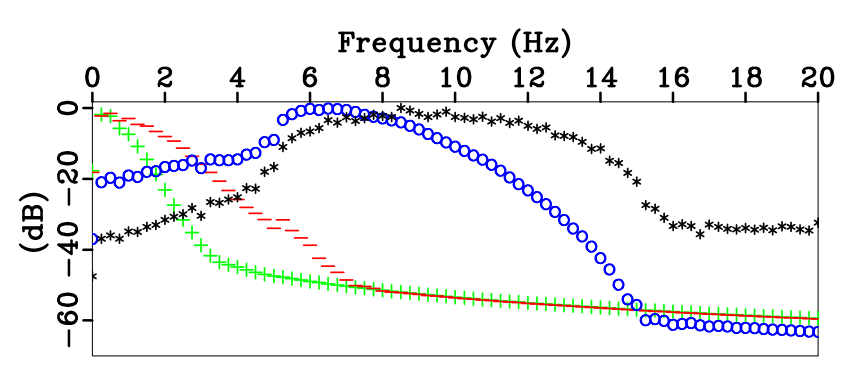

Figure 3. Average power spectrum (normalized) of shot gathers shown in Fig. 2. The marker symbols ' + ', '-', 'o' and ' $*$ ' represent the spectrum of the shot gathers obtained using $\xi=\{0.15,0.05,0.005,0\}$, respectively.

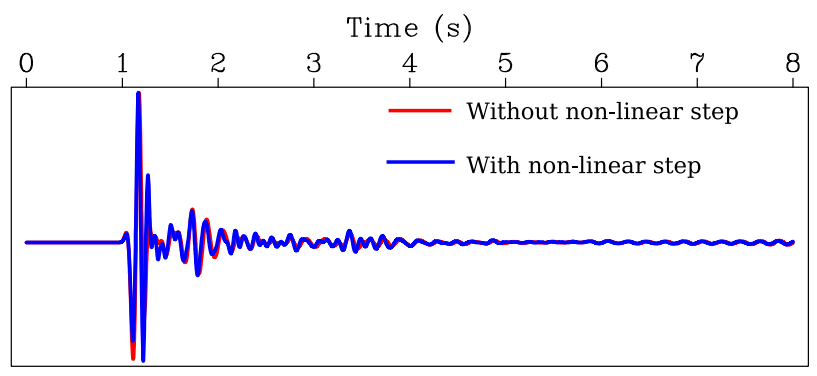

Figure 4. Overlay of seismic traces (normalized) with and without the nonlinear step in FCT $(\xi=0.005)$. They are kinematically almost similar.
Alkhalifah (2015) and Kazei et al. (2016) suggested the application of a scattering angle filter, as a preconditioner to the gradient of the objective function.

Almost all of the methods mentioned above present one or more of the following serious drawbacks:

(i) lacking robustness, especially with a complicated noisy field data set;

(ii) complicated to implement, using the existing framework of the available code;

(iii) computationally infeasible in large-scale 3-D problems.

However, all these methods follow the same protocol in their quest to reconstruct model parameters from seismic data by attempting to retrieve long-scale model wavelengths accurately, and then the short-scale wavelengths. This idea of an automated sequential retrieval of wavelengths through FWI has been contemplated since Mora (1989): the tomographic components of the model primarily governs the wave propagation, and the migration components attempt to focus scattering events in the tomographic model. Therefore, for an inversion process, it is quite natural to follow a hierarchical strategy aimed at retrieving long to short-scale components in a sequential manner. Also, a very common practice in FWI is to apply Gaussian kernel smoothing (Ravaut et al. 2004; Operto et al. 2006; Choi \& Alkhalifah 2018) or low pass filter (Sirgue et al. 2003; Sirgue \& Pratt 2004) on the gradient direction, before its application to update the background model. It is a post-processing step to the gradient evaluation, and it is very easy to implement. However, it is a static process and does not comply with the wave equation of propagation scheme at all. As a result, it guarantees no better outcomes from FWI. Moreover, FWI suffers from slow convergence 


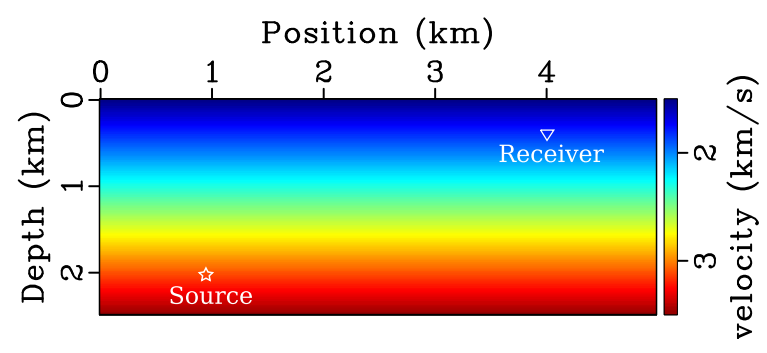

(a)

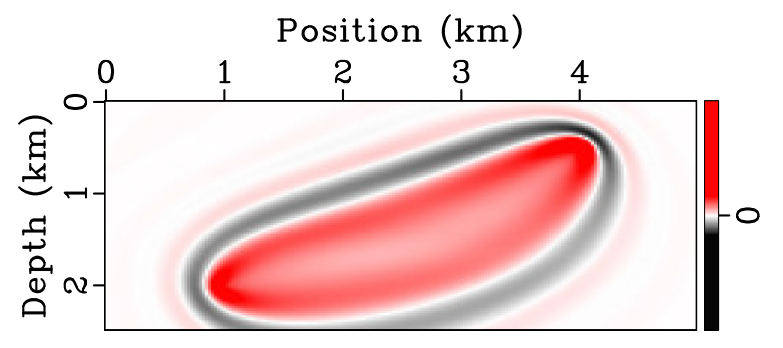

(c)

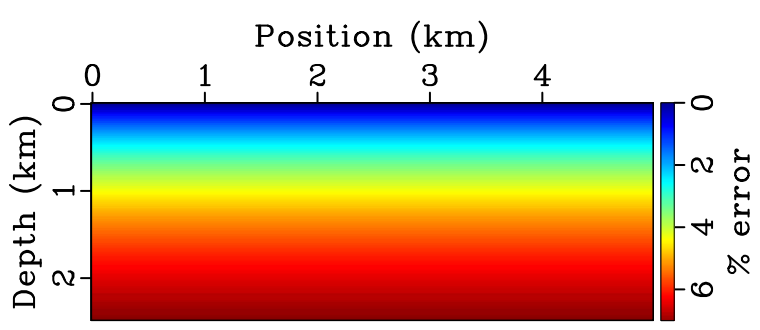

(b)

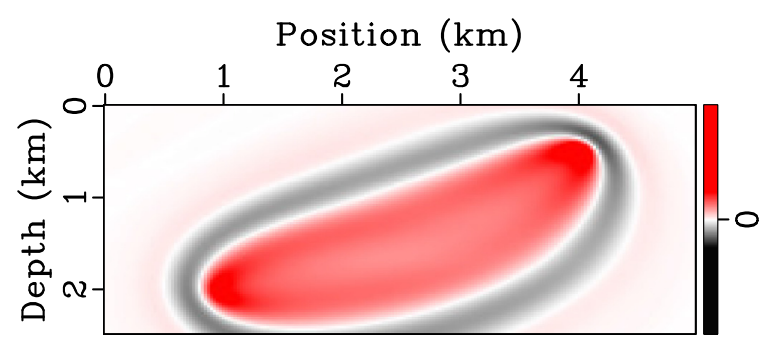

(d)

Figure 5. (a) True velocity model, (b) per cent error in the background velocity model of consideration, sensitivity kernels using (c) conventional method, (d) FCT method $(\xi=0.15)$ for a trace with $3 \mathrm{~Hz}$ dominant frequency. Source and receiver positions of the trace are also marked.

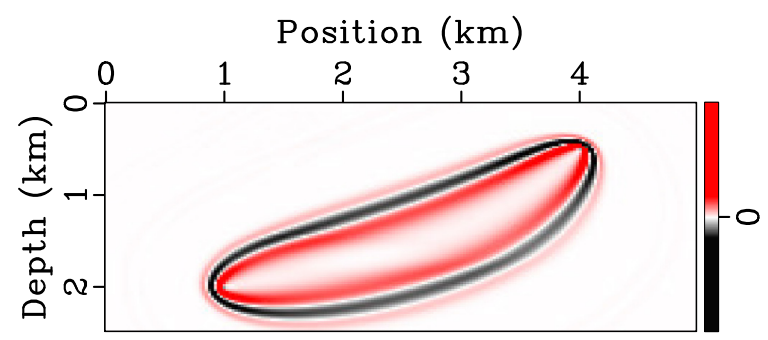

(a)

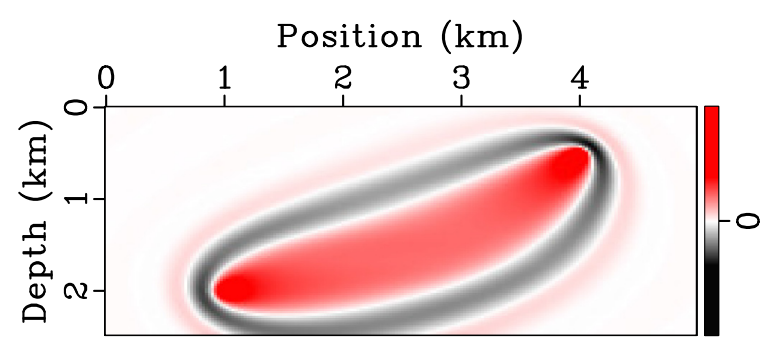

(b)

Figure 6. Sensitivity kernels for a trace of $10 \mathrm{~Hz}$ dominant frequency using (a) the conventional method and (b) the FCT method ( $\xi=0.15$ ).

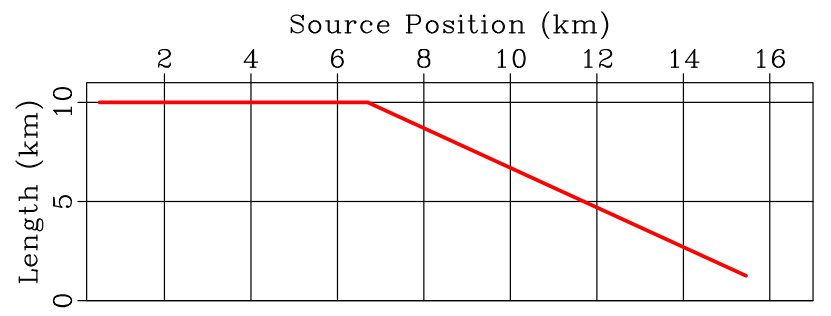

Figure 7. Available streamer length for each shot gather. Acquisition coverage towards the right part of the model is very poor.

rate in case of oversmoothing. Alkhalifah \& Choi (2016) and Xue et al. (2016) suggested the application of a smoothing kernel on seismic records, in order to promote sufficient smoothness in the gradient evaluation. However, like Gaussian smoothing, it is very sensitive to the kernel-size of consideration.
In order to overcome most of the shortcomings mentioned above, we introduce a multiscale flux-corrected transport (FCT) algorithm which uses two finite difference schemes: a transport and a diffusion. The diffusion flux at a gridpoint is governed by the solution of the wave equation, that is the wavefield, and a smoothing parameter that controls the hierarchical strategy we are advocating in this paper. In doing so, we observe a couple of benefits associated with this method. First, it yields a smooth gradient at the earlier iterations of FWI by promoting the lower frequency content of the seismic data. Secondly, it is easily compatible with the existing FWI code, with any objective function. However, although promising, this method is not fully immune to cycle-skipping, for any arbitrary starting model and high frequency of the data set.

There is very little literature on FCT in the seismic industry (Fei \& Larner 1995; Zheng et al. 2006); however, it is very common practice in computational fluid dynamics (CFD, Boris \& Book 1973; 


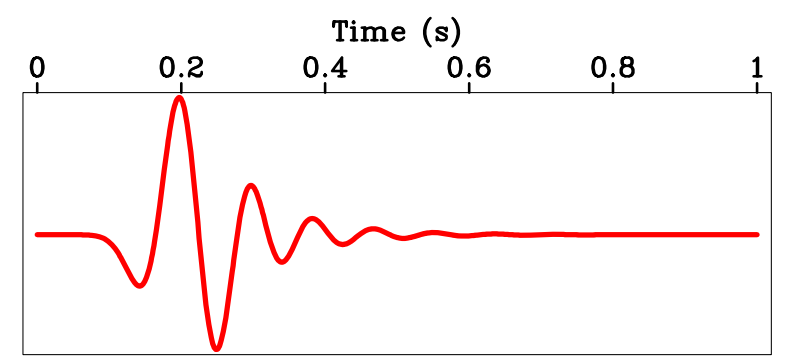

(a)

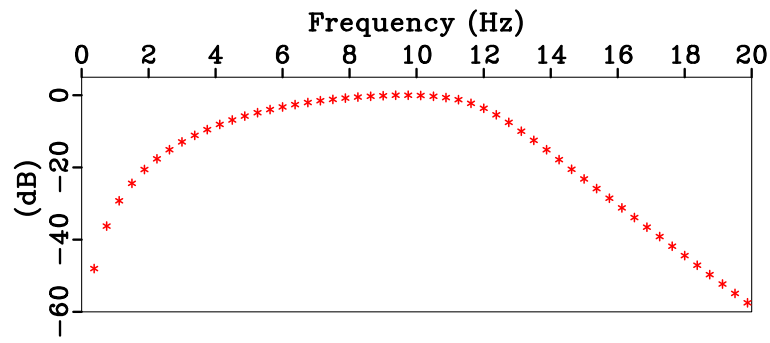

(b)

Figure 8. (a) A Ricker wavelet of dominant frequency at $10 \mathrm{~Hz}$ filtered by a high-cut filter at $13.0 \mathrm{~Hz}$ is used as a source-function, (b) its power spectrum (normalized).

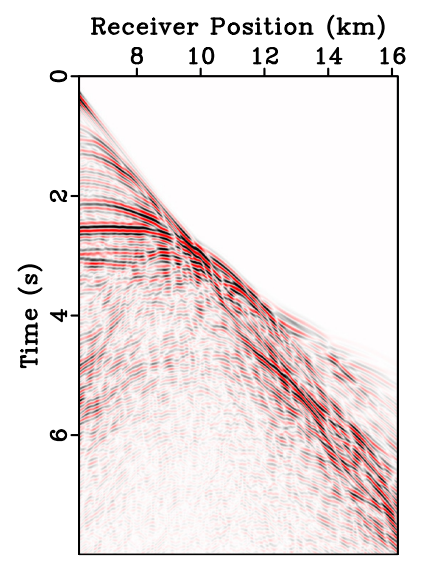

(a) (b)

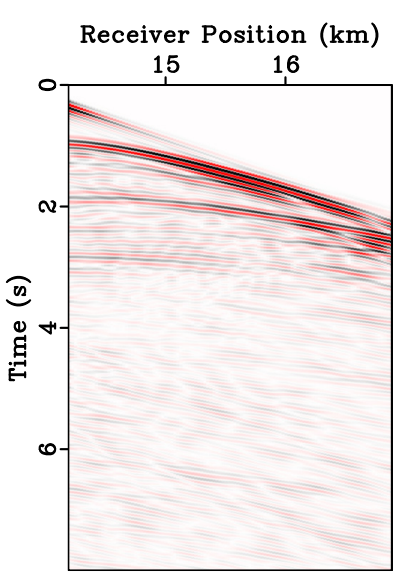

Figure 9. Shot gathers from the sources placed at (a) $x=6.0 \mathrm{~km}$ and (b) $x$ $=14.0 \mathrm{~km}$.

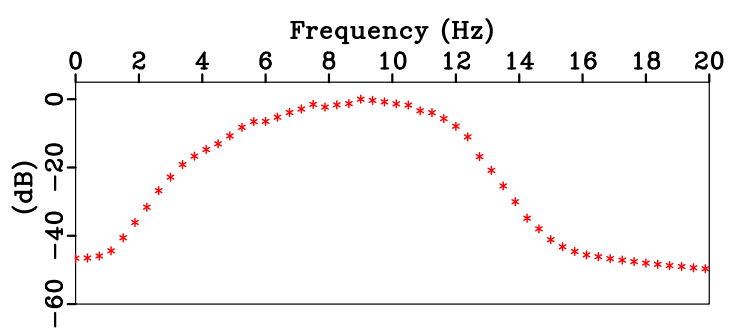

Figure 10. Average power spectrum (normalized) of the shot gather shown in Fig. 9(a).

Book et al. 1975; Boris \& Book 1976; Zalesak 1979) in order to remove the inviscid shocks and steep gradients near discontinuities. In addition to the transport and diffusion steps, it involves a third step to remove the undue flux corrections from the shock-profile. This third limiting step is very non-linear in nature although carried out based on the principal of mass conservation (Boris \& Book 1997). For the first time, Fei (1994) and Fei \& Larner (1995) reported FCT in seismic modelling and revere-time migration (RTM). The goal of their work was to provide a computationally feasible algorithm in order to obtain a high-resolution RTM image without numerical dispersion. A high-resolution RTM image requires fine numerical spatial grids. As a consequence of the Courant-Friedrichs-Lewy (CFL) condition, the fine spatial grids require the wavefield to be extrapolated using small time increments. Moreover, a detailed description of the image, especially for the deep regions, requires the use of the entire data, at least as much as possible. However, with the improvements in computational power, mitigation of numerical dispersion in RTM image is no longer as challenging as it used to be. $^{2}$ Here in this article, we push the barrier to utilize FCT from forward modelling to adjoint-state methods and in particular FWI. In doing so, we compromise with the non-linear step involved in Fei \& Larner (1995).

The outline of the rest of this paper is as follows. We begin the theory section with a short review of FCT in CFD. Next, we describe its application in exploration seismology as shown in Fei \& Larner (1995) and the necessary modification to make FCT compatible for adjoint-state methods. We also show the role of smoothing parameter $\xi$ in the context of promoting low frequencies from a source of high-frequency dominant seismic data. Towards that aim, we show the nature of the sensitivity kernel of FWI using FCT. In order to demonstrate the versatility of our proposed method, we consider a synthetic, noise-free data set obtained from the Marmousi II model. The model is well reconstructed starting from a poor initial 1-D model whose velocity increases linearly with depth. Also, we demonstrate its application on a noisy data set from Marmousi II model. Finally, we show an application to a field data set from offshore Australia.

\section{THEORY}

$\mathbf{L}(\mathbf{m}) \mathbf{u}=\mathbf{s}$,

where $\mathbf{m}$ describes the discretized earth subsurface parameters defined at all space points, $\mathbf{s}$ represents the seismic source defined at all time samples and the space points in the model, and $\mathbf{L}$ underpins the governing physics of wave propagation in order to extrapolate

\footnotetext{
${ }^{2}$ The computational requirements for FWI of a 3-D data set that are collected using modern broad-band, long offset, full-azimuthal systems are enormous.
} 


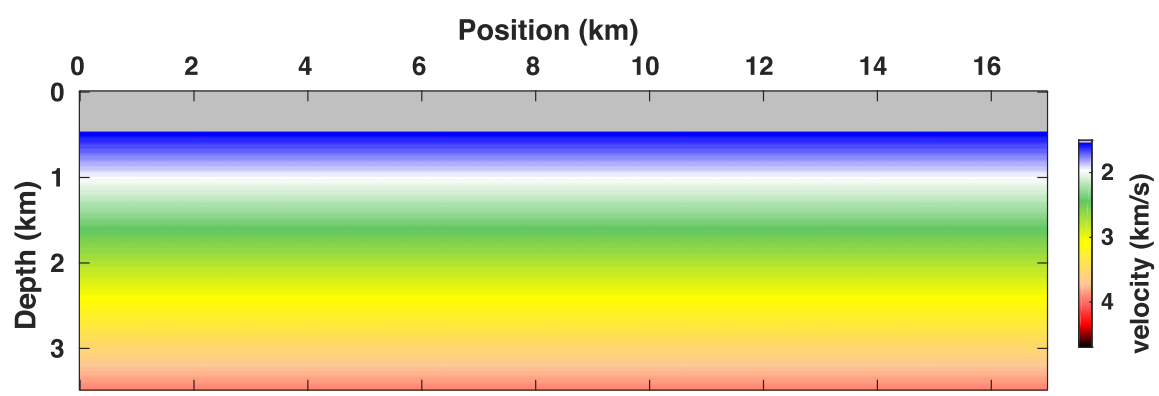

Figure 11. 1-D starting model. Its velocity increases linearly with depth from the water bottom.

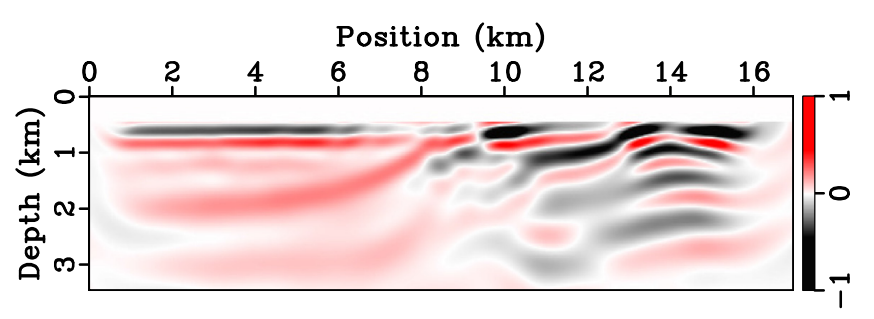

(a)

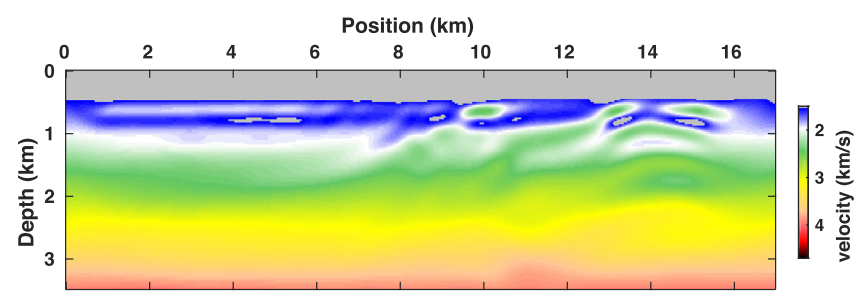

(b)

Figure 12. (a) Gradient direction of FWI with $\xi=0.20$ at first iteration and (b) the model after the update process.

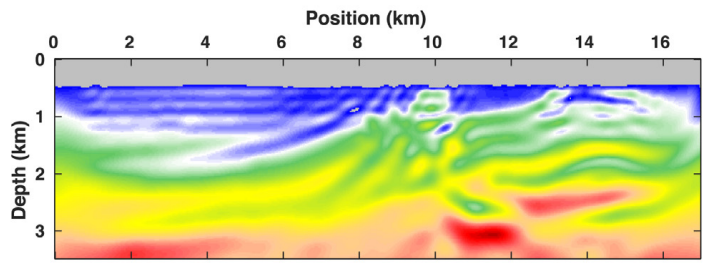

(a)

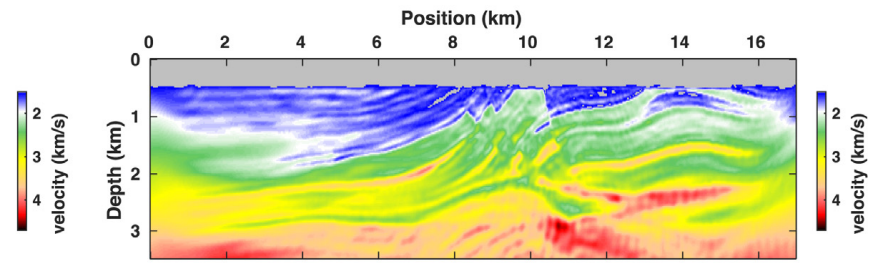

(b)

Figure 13. Evolution of velocity model through FWI using FCT algorithm at (a) 32nd iteration ( $\xi=0.05$ ), (b) 51st iteration ( $\xi=0.001$ ).

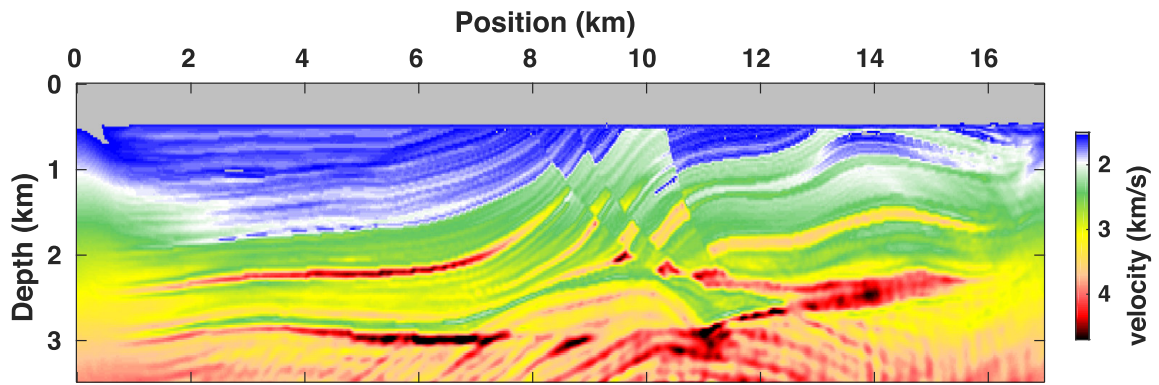

Figure 14. Final inverted model using FCT. Compare with the true model shown in Fig. 1. Despite limitations such as poor acquisition logistics into account, FCT retrieves most of the model. 

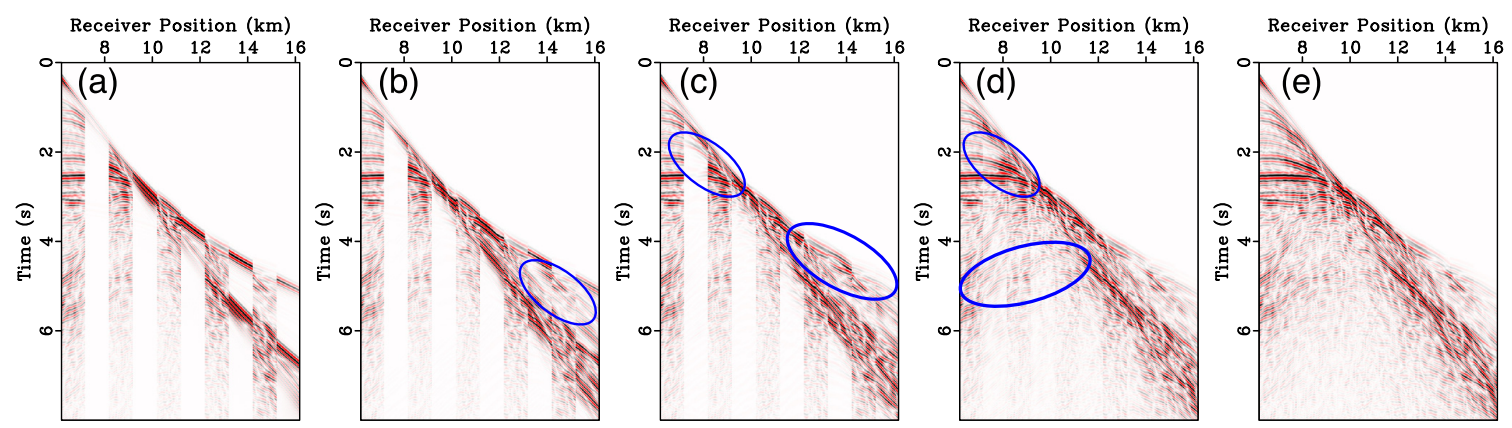

Figure 15. Seismic data matching from the source, at $x=6.0 \mathrm{~km}$, by interleaving 40 traces, starting from the observed followed by predicted traces. From left to right predicted data synthesized at FWI iterations of 1,2,33, 52 and 81, respectively. Predicted data at the first iteration suffers from cycle-skipping. Over the iterations, matching of observed and predicted data improves as indicated by ellipses. At the final iteration, the predicted data is almost identical with the observed ones.

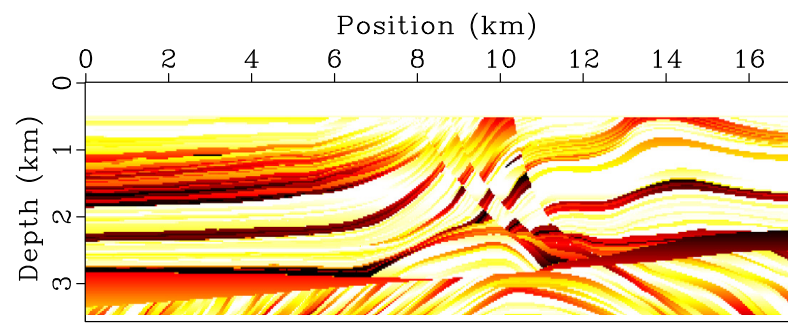

(a)

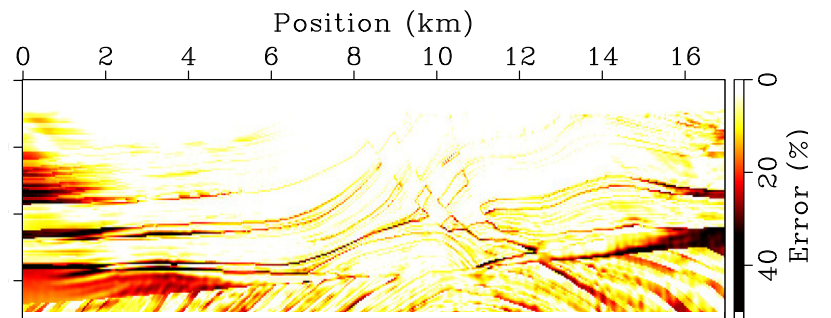

(b)

Figure 16. Per cent error of (a) initial velocity model, (b) final velocity model. The inversion process retrieves most of the velocity parameters, especially at the shallow depth. Due to the limitations in acquisition sampling and the finite frequency data set, inversion can not recover the velocity field at deep and the sharp boundaries.

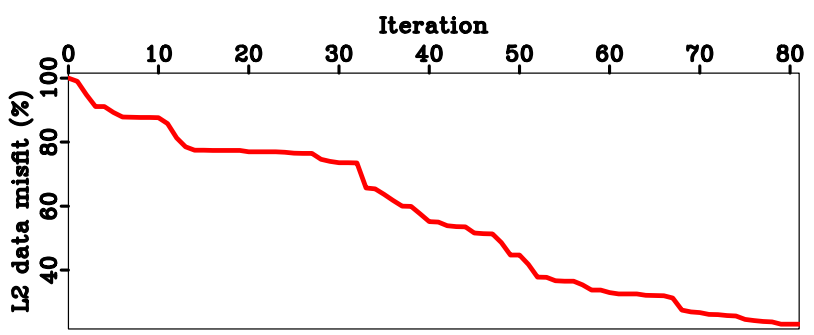

Figure 17. FCT-based FWI reduced the misfit by almost 75 percent. We assigned $\xi=\{0.2,0.15,0.1,0.05,0.01,0.008,0.005,0.001,0\}$ over the interations of the multiscale approach. The first stage $(\xi=0.2)$ took 4 iterations followed by $\{6,4,4,7,7,5,12,31\}$ number of iterations for the rest of $\xi$ values to retrieve the final velocity model shown in Fig. 14.

the wavefield $\mathbf{u}$ at all the space points in the model and time samples. In general, $\mathbf{L}$ is a finite difference time-stepping explicit implementation of the appropriate wave equation. Here, we advocate the modification of $\mathbf{L}$ using FCT, in order to embrace a multiscale approach in FWI and obtain the final inverted model. Before developing the adjoint-state formulation to FCT algorithms for FWI, we explore FCT algorithms on CFD and the seismic industry in Sections 2.1 and 2.2, respectively.

\subsection{FCT in CFD}

FCT is a popular technique in CFD (Zalesak 1979; Löhner et al. 1987; Tóth \& Odstrčil 1996) to remove the non-physical numerical oscillations from the mass density $(\rho)$ profile by honouring the mass-conserving property of the continuity equation, given by:

$\frac{\partial \rho}{\partial t}+\nabla \cdot(\rho v)=0$

where $v$ is a given velocity field. It relaxes strict asymptotic ordering expansions with the much more flexible physical fact that positivity must be maintained in the vicinity of strong gradients, in order to limit the non-physical behavior of a conserved quantity, that is mass density. In doing so, the process involves two steps : a conventional finite difference transport, followed by a local corrective diffusion to the region where non-physical ripples tend to form. Since there is no a priori knowledge about the region of non-physical ripples, the practical implementation of FCT requires to supply sufficient diffusion everywhere, followed by a limitation of the flux wherever over correction happens in a non-linear way. The flux limitation at the evolution of $\rho$, although non-linear because of its spatial dependency on $\rho$, confirms the absence of accentuation of already existing extrema points on the profile (Boris \& Book 1976).

The practical implementation of FCT involves two linear operations: a transport $(T)$ and a diffusion $(D)$, followed by a non-linear operation of antidiffusion $(A)$ to limit the flux. The old values of the density profile, $\left\{\rho_{j}^{0}\right\}$, are transported to by an operation $(I+T)$. They are subsequently subjected to the diffusion process in order to obtain $\left\{\rho_{j}^{T D}\right\}$. After the antidiffusion, the values at the new time step form the flux-corrected density profile, $\left\{\rho_{j}^{1}\right\}$. Symbolically, the explicit version of FCT can be given by:

$$
\begin{aligned}
\rho^{T D} & =(I+T+D) \rho^{0}, \\
\rho^{1} & =(I+A) \rho^{T D}=(I+A)(I+T+D) \rho^{0} .
\end{aligned}
$$




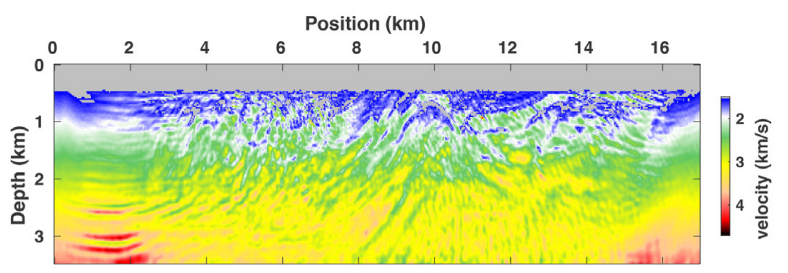

(a)

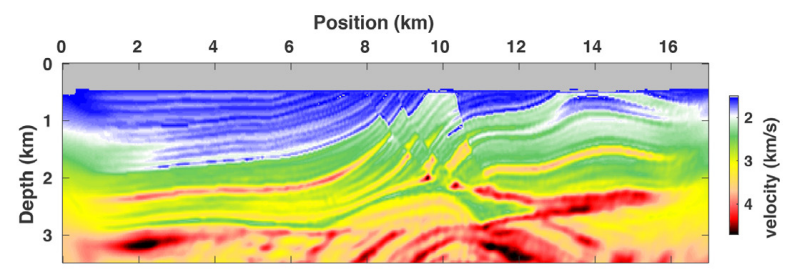

(b)

Figure 18. Final inverted model using (a) conventional method for the full bandwidth and (b) multiscale method as described in Bunks et al. (1995). In the multiscale approach, we inverted for sub data sets of frequencies less than $1 \mathrm{~Hz}$, followed by $2 \mathrm{~Hz}, 4 \mathrm{~Hz}, 10 \mathrm{~Hz}$ and the full bandwidth.

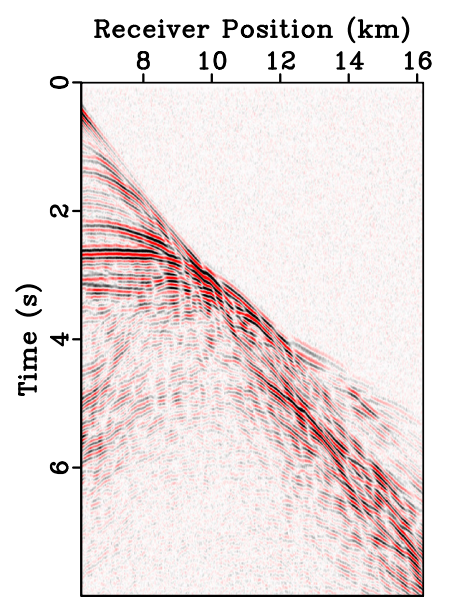

(a)

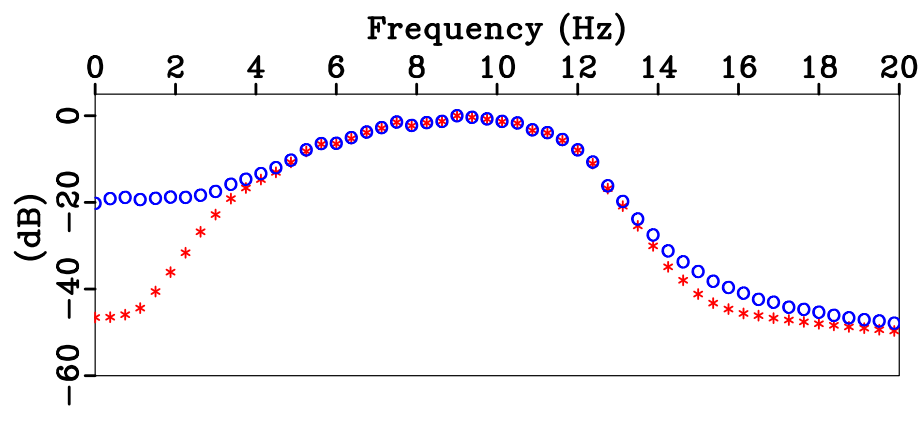

(b)

Figure 19. (a) Noise is added to the shot gather shown in Fig. 9(a) to prepare noisy data, and (b) its normalized power spectrum (marker symbol 'o') compared with noise-free-data (marker symbol ' $*$ ').

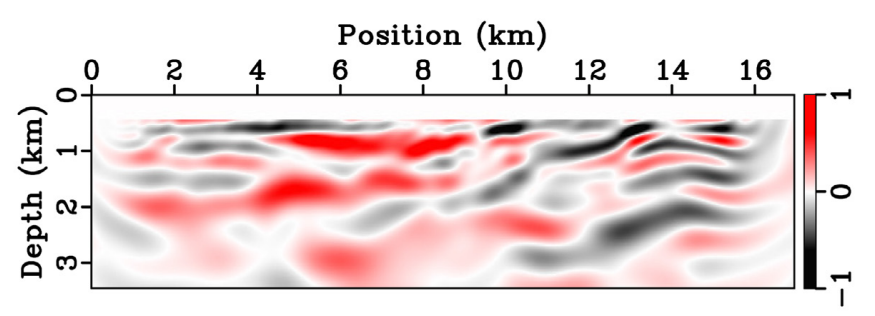

(a)

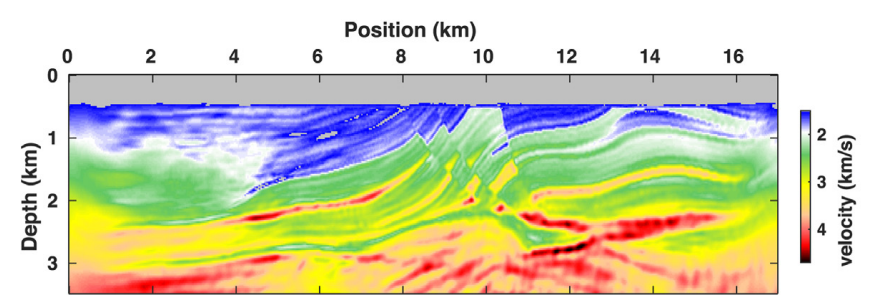

(b)

Figure 20. (a) Gradient direction of FWI with $\xi=0.15$ at first iteration and (b) final inverted model using FCT.

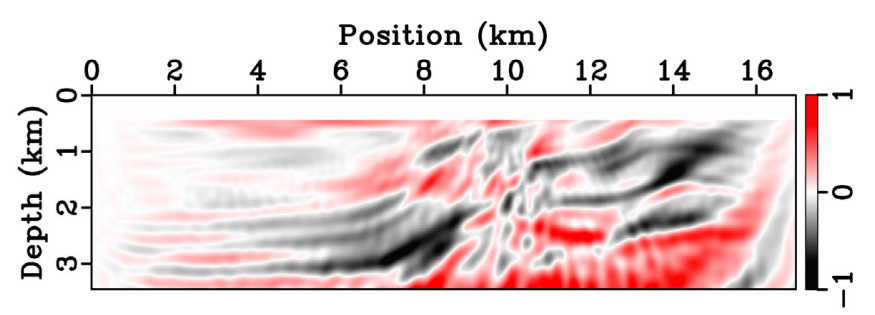

(a)

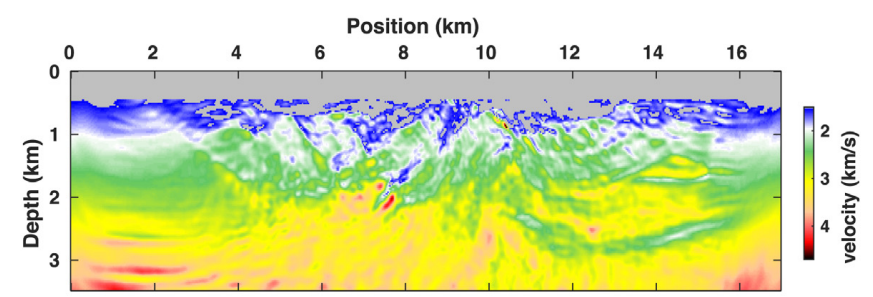

(b)

Figure 21. (a) Gradient direction of FWI at first iteration and (b) final inverted model using conventional multiscale approach. 


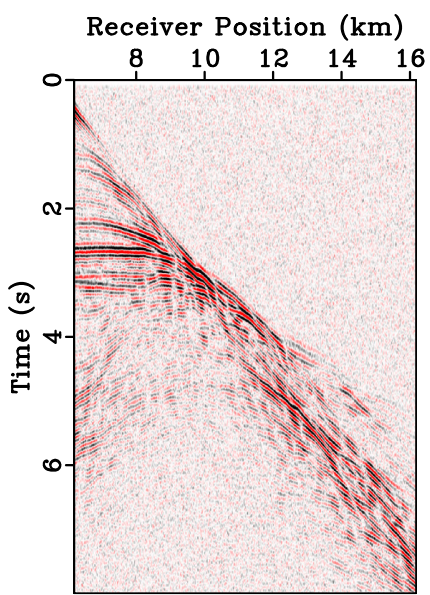

(a)

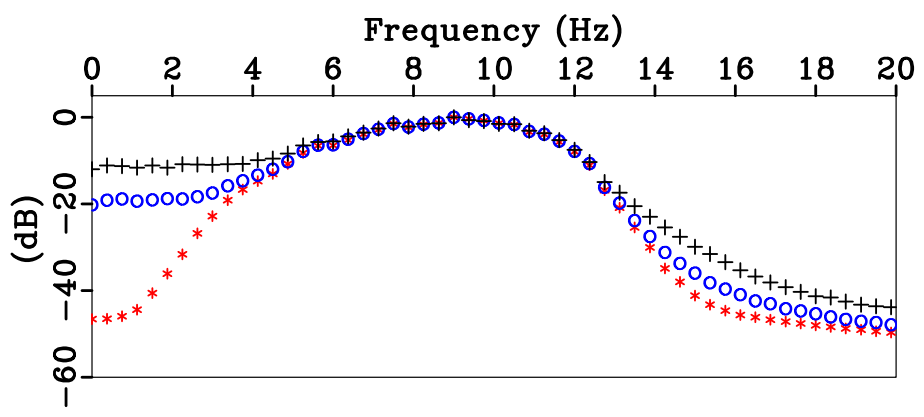

(b)

Figure 22. Shot gathers from the sources placed at (a) $x=6.0 \mathrm{~km}$ and its (b) normalized power spectrum (marker symbol '+') compared with those from the previous two examples: marker symbols 'o' for previous noisy-data and ' $*$ ' for noise-free-data.

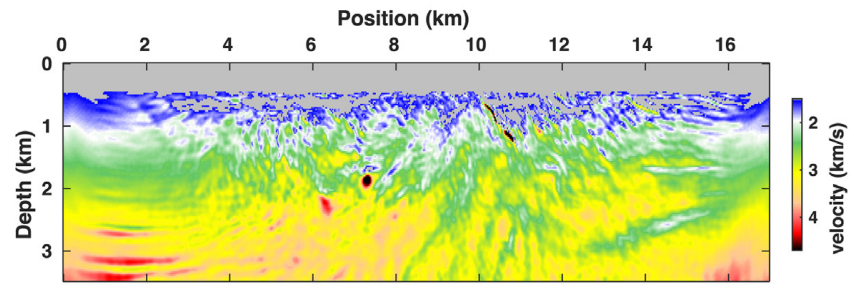

(a)

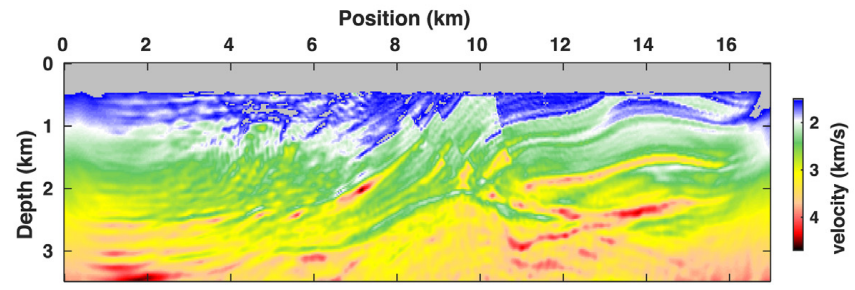

(b)

Figure 23. Final inverted model of the noisy data displayed in Fig. 22(a) using (a) conventional multiscale method and (b) FCT algorithm. Apart from the left part of the model, FCT-based FWI recovers the velocity reasonably well, especially the shallow region of the model.

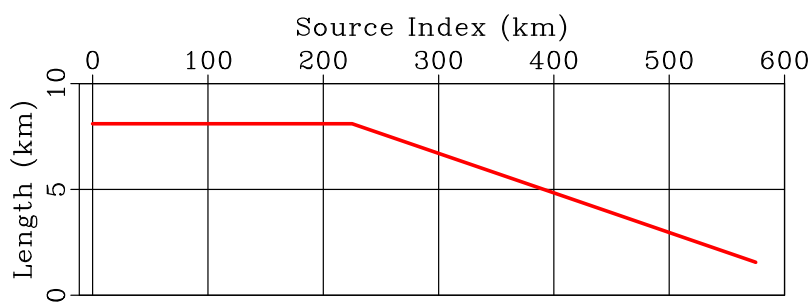

Figure 24. Available streamer length for the shots of consideration in the field-data example.

Depending on the numerical-scheme of consideration in $T$, the implementation of $D$ and $A$ varies. More Details are given in Boris \& Book (1976); Zalesak (1979). Inspired by its success on CFD, Fei \& Larner (1995) applied FCT in exploration seismology for the first time, with a goal of circumventing numerical dispersion, even with coarse grid samples. Clearly, the problems of numerical dispersion can easily be negotiated by sampling the wavefield at finer grid intervals. The advancements in computational power make finer grid affordable, which might be the primary reason for which FCT is not so popular among the seismic community. Another shortcoming of FCT is that it requires the selection of two parameters $(\xi, \psi)$ for identifying the numerically affected troublesome regions. However, we can exploit this phenomenon to steer FCT in FWI to reconstruct the unknown parameters from seismic data.

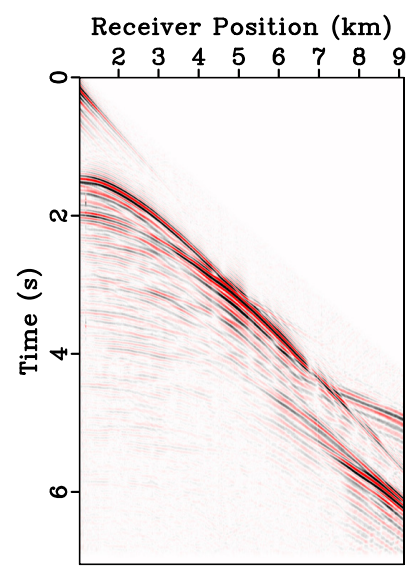

(a)

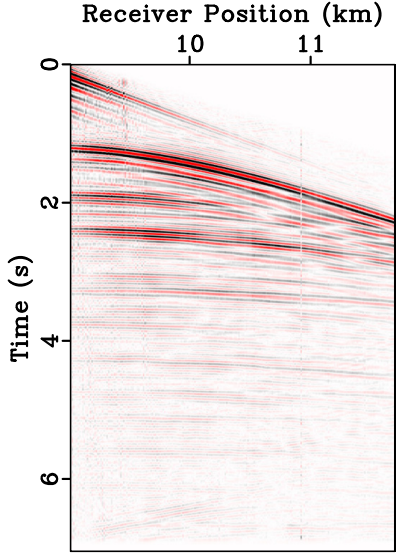

(b)
Figure 25. Shot gathers from the sources placed at (a) $x=1.0 \mathrm{~km}$ and (b) $x=9.0 \mathrm{~km}$.

\subsection{Forward modelling using FCT}

This section describes FCT in the context seismic wavefield extrapolation as proposed in Fei \& Larner (1995), and also the necessary modifications required to make it viable for the adjoint-state method. 


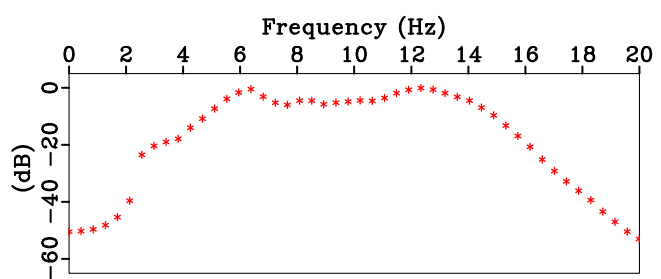

Figure 26. Average frequency content (normalized) of the shot gather displayed in Fig. 25(a).

Without loss of generality, we consider the 2nd-order seismic wave equation in a 2-D acoustic medium to describe the methodology of the FCT technique. We denote the wavefield at any arbitrary gridpoint $(i, j)$ with $u_{i, j}^{n}$ for any arbitrary $n$th time-step. Its numerical flux is defined as $f_{i+1 / 2, j}^{n}=u_{i+1, j}^{n}-u_{i, j}^{n}$ and $f_{i, j+1 / 2}^{n}=u_{i, j+1}^{n}-u_{i, j}^{n}$ along the $x$ and $z$ directions, respectively. Next, we compute the associated diffusive flux defined as:

$$
\begin{aligned}
q_{i+1 / 2, j}^{n-1 / 2} & =\xi\left(f_{i+1 / 2, j}^{n}-f_{i+1 / 2, j}^{n-1}\right) \\
& =\xi\left(f_{i, j+1 / 2}^{n}-f_{i, j+1 / 2}^{n-1}\right) ; \quad 0 \leq \xi \leq 1 .
\end{aligned}
$$

Therefore the diffusion flux of $u$ is evaluated by employing two finite-difference operations: a spatial derivative operation followed by a temporal one. Using eq. (4), we modify the wavefield at $(n+$ 1)th time step as follows:

$\tilde{u}_{i, j}^{n+1}=u_{i, j}^{n+1}+\left(q_{i+1 / 2, j}^{n-1 / 2}-q_{i-1 / 2, j}^{n-1 / 2}\right)+\left(q_{i, j+1 / 2}^{n-1 / 2}-q_{i, j-1 / 2}^{n-1 / 2}\right)$.

$\tilde{u}$ is used for its propagation to the next time-step. Thus, the wavefield extrapolation benefits from an addition of a small corrective quantity (a function of both $\mathbf{x}$ and $t$ ) at every time step, yielding a smooth solution with a loss in amplitude. The corrective measure is carried out in a conservative way (except at the boundaries), that is whenever a quantity of diffusive flux is added at one point, that same amount is subtracted from somewhere else. Thus, it is moved from point to point locally, but with no net loss or gain to the system. However, the smoothness order depends on the parameter $\xi$, which varies with the scheme of finite difference under consideration. $\xi$ should not be too big to violate the CFL criteria. In order to depict the role of $\xi$ in seismic modeling, we show the wavefield recorded at a depth of $z=2.0 \mathrm{~km}$ of Marmousi II model (Fig. 1) from a source placed at $(x, z)=(8.0,0) \mathrm{km}$ (Fig. 2). In Fig. 2, we observe that large $\xi$ yields a smoother wavefield compared to the conventional counterpart $(\xi=0)$. Fig. 3 displays the average power spectra of the shot gathers shown in Fig. 2.

Eq. (5) represents the second step of FCT, that is an uncontrolled diffusion process performed everywhere in the model, and responsible for smoothing the wavefield. In order to prevent the diffusion fluxes from overcorrection, FCT requires the use of an antidiffusion strategy consisting of the following cumbersome steps:

- Compute the diffusive fluxes at time step $n+1$ :

$$
\begin{array}{rlrl}
\tilde{q}_{i+1 / 2, j}^{n+1 / 2} & =\psi\left(f_{i+1 / 2, j}^{n+1}-f_{i+1 / 2, j}^{n}\right) & \tilde{q}_{i, j+1 / 2}^{n+1 / 2} \\
& =\psi\left(f_{i, j+1 / 2}^{n+1}-f_{i, j+1 / 2}^{n}\right) ; \quad 0 \leq \psi \leq 1 .
\end{array}
$$

- Compute the following quantities using eq. (5):

$$
\begin{aligned}
X_{i+1 / 2, j} & =\left(\tilde{u}_{i+1, j}^{n+1}-u_{i+1, j}^{n}\right)-\left(\tilde{u}_{i, j}^{n+1}-u_{i, j}^{n}\right) \quad Y_{i, j+1 / 2} \\
& =\left(\tilde{u}_{i, j+1}^{n+1}-u_{i, j+1}^{n}\right)-\left(\tilde{u}_{i, j}^{n+1}-u_{i, j}^{n}\right) .
\end{aligned}
$$

- Compute the following using eqs (6) and (7):

$$
\begin{aligned}
X_{i+1 / 2, j}^{c} & =S_{x} \max \left\{0, \min \left[S_{x} X_{i-1 / 2, j} \text { abs }\left(\tilde{q}_{i+1 / 2, j}^{n+1}\right), S_{x} X_{i+3 / 2, j}\right]\right\} \\
Y_{i+1 / 2, j}^{c} & =S_{y} \max \left\{0, \min \left[S_{y} Y_{i, j-1 / 2} \operatorname{abs}\left(\tilde{q}_{i, j+1 / 2}^{n+1}\right), S_{y} Y_{i, j+3 / 2}\right]\right\} \\
S_{x} & =\operatorname{sign}\left(\tilde{q}_{i+1 / 2, j}^{n+1 / 2}\right) \\
S_{y} & =\operatorname{sign}\left(\tilde{q}_{i, j+1 / 2}^{n+1 / 2}\right)
\end{aligned}
$$

- Correct the eq. (5) using eq. (8):

$u_{i, j}^{n+1}=\tilde{u}_{i, j}^{n+1}+\left(X_{i+1 / 2, j}^{c}-X_{i-1 / 2, j}^{c}\right)+\left(Y_{i, j+1 / 2}^{c}-X_{i, j-1 / 2}^{c}\right)$.

Eq. (9) represents the flux-corrected seismic wavefield (Fei \& Larner 1995). However, we exclude the essential limiting step from FCT with the goal of obtaining the FWI gradient. The primary reasons behind this exclusion are:

- It is highly non-linear. Eq. (8) involves non differentiable operators such as maximum, minimum, absolute and signum.

- Seismic source wavelet being continuous and simple in nature is very unlikely to yield very strong ripples like shock waves.

Therefore, our FCT implementation halts at the second stage (eq. 5) of the original version (eq. 9). However, this circumvention does not affect the wavefield as such, particularly in the context of smoothing the wavefield. For comparison purposes, we consider a trace recorded at $z=2.0$ from the example given in Fig. 2. We show that FCT leads an almost identical solution with or without the non-linear step (Fig. 4). Also, the absence of a non-linear step effectively makes FCT's algorithm very easy to implement with conventional FWI machinery.

A further simplification of eq. (5) enables us to formulate the FCT on a matrix-vector form, as follows:

$\tilde{\mathbf{u}}^{n+1}=\mathbf{u}^{n+1}+D\left(\mathbf{u}^{n}-\mathbf{u}^{n-1}\right) \quad$ with $\quad D=\xi\left(\begin{array}{ccc}0 & -1 & 0 \\ -1 & 4 & 1 \\ 0 & 1 & 0\end{array}\right)$

where the superscript $n>1$ is a time step; $\tilde{\mathbf{u}}$ is the flux-corrected wavefield that yields the transport solution $\mathbf{u}$ for the next time-step. The $3 \times 3$ kernel $D$ is a self-adjoint linear operation due to ignoring the flux's limitations. In a nutshell, the FCT operator $\tilde{L}$ is simply the wave equation operator $L$ followed by $D$.

\subsection{Multiscale FWI using FCT}

FWI is a non-linear data-matching technique aiming to achieve a detailed estimate of the subsurface model parameters from seismic data. Given an initial model, the predicted data $\left(\mathbf{d}_{\text {mod }}\right)$ satisfy the wave eq. (1) at the receiver positions of the experiment. Mathematically, $\mathbf{d}_{\text {mod }}=R \mathbf{u}$ where $R$ is a detection operator to extract $\mathbf{u}$ only at the receiver positions. The next step involves updating the initial model in order to reduce the misfit $(f)$ between the observed $\left(\mathbf{d}_{o b s}\right)$ and the predicted seismic data, and, feed back the updated model into the iterative procedure as the background model. This iterative loop is carried out until the data-misfit is sufficiently small. At every iteration, the background model is updated by a search direction (i.e. a function of the gradient of the misfit), depending on the choice of optimization algorithms. For instance, the search direction in the method of steepest descent is given by the negative gradient of the misfit function. Following the adjoint-state method (Plessix 2006), the gradient computation of FWI misfit function of 

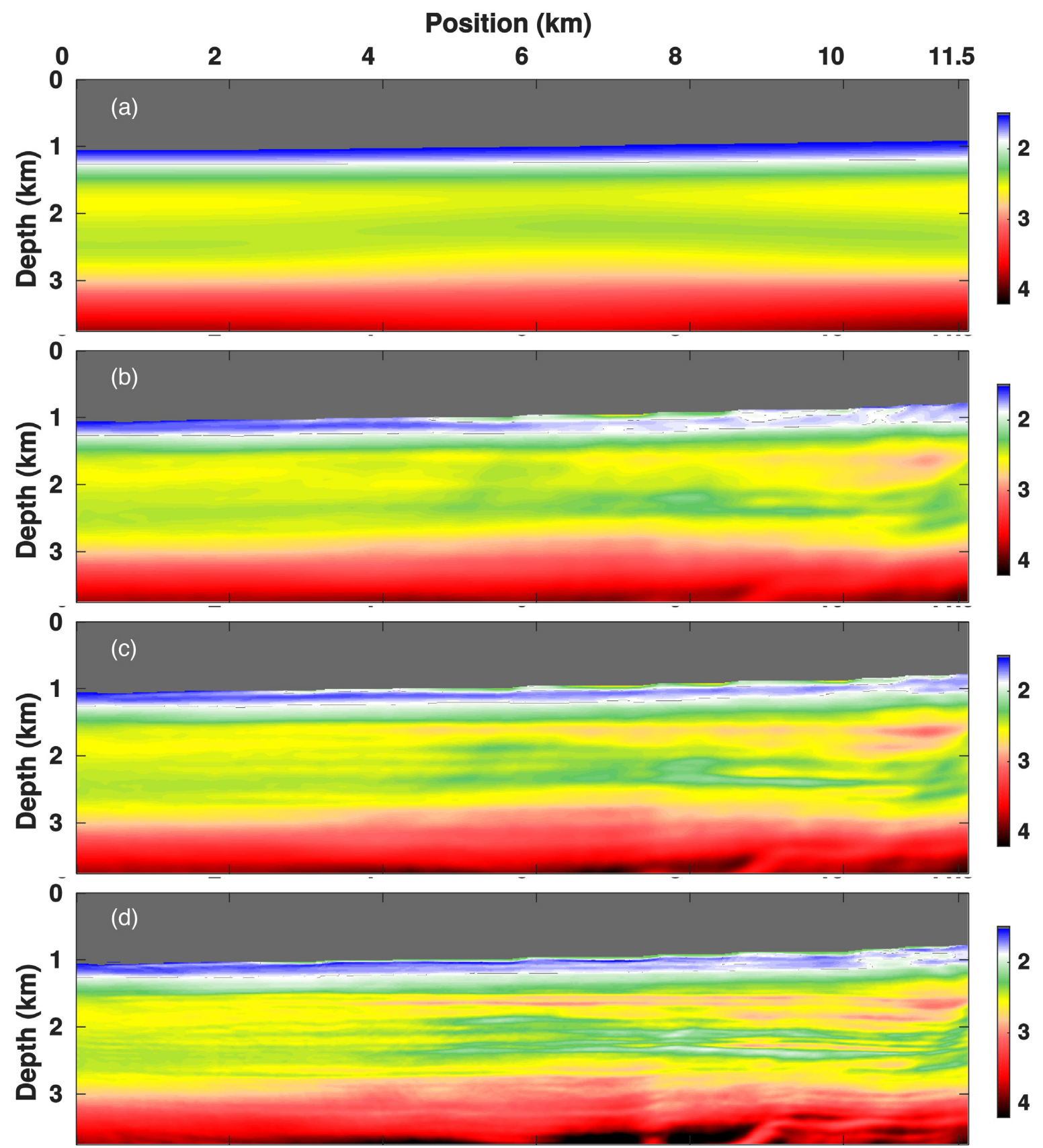

Figure 27. (From top to bottom) Evolution of the velocity model through FWI using FCT approach. The top panel shows the starting model, followed by two intermediate results of inversion process. The bottom panel displays the final inverted velocity model using FCT.

the form $f=\frac{1}{2} \mathbf{y}^{T} \mathbf{y}$ is given by:

$\frac{\partial f}{\partial \mathbf{m}}=-\left(\frac{\partial \mathbf{L}}{\partial \mathbf{m}} \mathbf{u}\right)^{T}\left(\mathbf{L}^{-1}\right)^{T} R^{T} \delta \mathbf{s}$,

where $\delta \mathbf{s}={\frac{\partial \mathbf{y}}{\partial \mathbf{d}_{\text {mod }}}}^{T} \mathbf{y}$ is known as the adjoint-source; $\mathbf{y}$ is a function of $\mathbf{d}_{\text {mod }}$ and $\mathbf{d}_{\text {obs }}$ (Lailly 1983; Pratt et al. 1998). Eq. (11) states (from right to left) that the adjoint source from the position of receivers $\left(R^{T} \delta \mathbf{s}\right)$ is to be back-propagated in time, using an operator $\mathbf{L}^{T}$, to perform a temporal cross-correlation with the forward wavefield (u) that was affected by an additional operator $\frac{\partial \mathbf{L}}{\partial \mathbf{m}}$, in order to evaluate the gradient of an objective function of the form $f$. In particular, $\mathbf{y}$

$$
\begin{aligned}
& =\mathbf{d}_{m o d}-\mathbf{d}_{o b s} \text { implies } \delta \mathbf{s}=\mathbf{d}_{m o d}-\mathbf{d}_{o b s}, \text { and } \\
& \begin{array}{l}
\frac{\partial f}{\partial \mathbf{m}}=-\left(\frac{\partial \mathbf{L}}{\partial \mathbf{m}} \mathbf{u}\right)^{T}\left(\mathbf{L}^{-1}\right)^{T} R^{T}\left(\mathbf{d}_{m o d}-\mathbf{d}_{o b s}\right) \\
\quad=2 \mathbf{m}_{b}<\nabla^{2} \mathbf{u}, \mathbf{v}>\quad \text { with } \quad \mathbf{L u}=f \quad \& \quad \mathbf{L}^{T} \mathbf{v}=\mathbf{d}_{m o d}-\mathbf{d}_{o b s},
\end{array}
\end{aligned}
$$

where $<,>$ denotes the dot product between two vectors (the timeseries, in our case), $\mathbf{m}_{b}$ is the background velocity at the gridpoint $\mathbf{x}$.

As mentioned earlier, The FCT modelling operator is nothing but a modification in the wave-equation engine. Therefore, a formulation of the gradient direction, at a gridpoint $\mathbf{x}$, using FCT, $\tilde{\mathbf{g}}$, also 


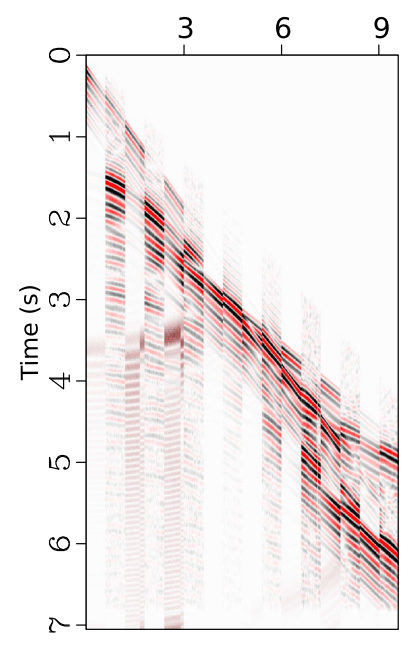

(a)

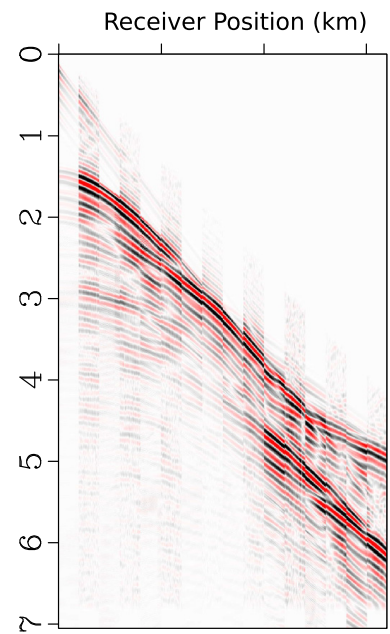

(b)
Figure 28. Shot gather displaying interleaved predicted and observed data using the (a) initial velocity and (b) final velocity model. We intersperse 40 traces starting form the predicted followed by observed data. Note that the final inverted velocity model predicts a data set that match the observed ones better than the initial model does.
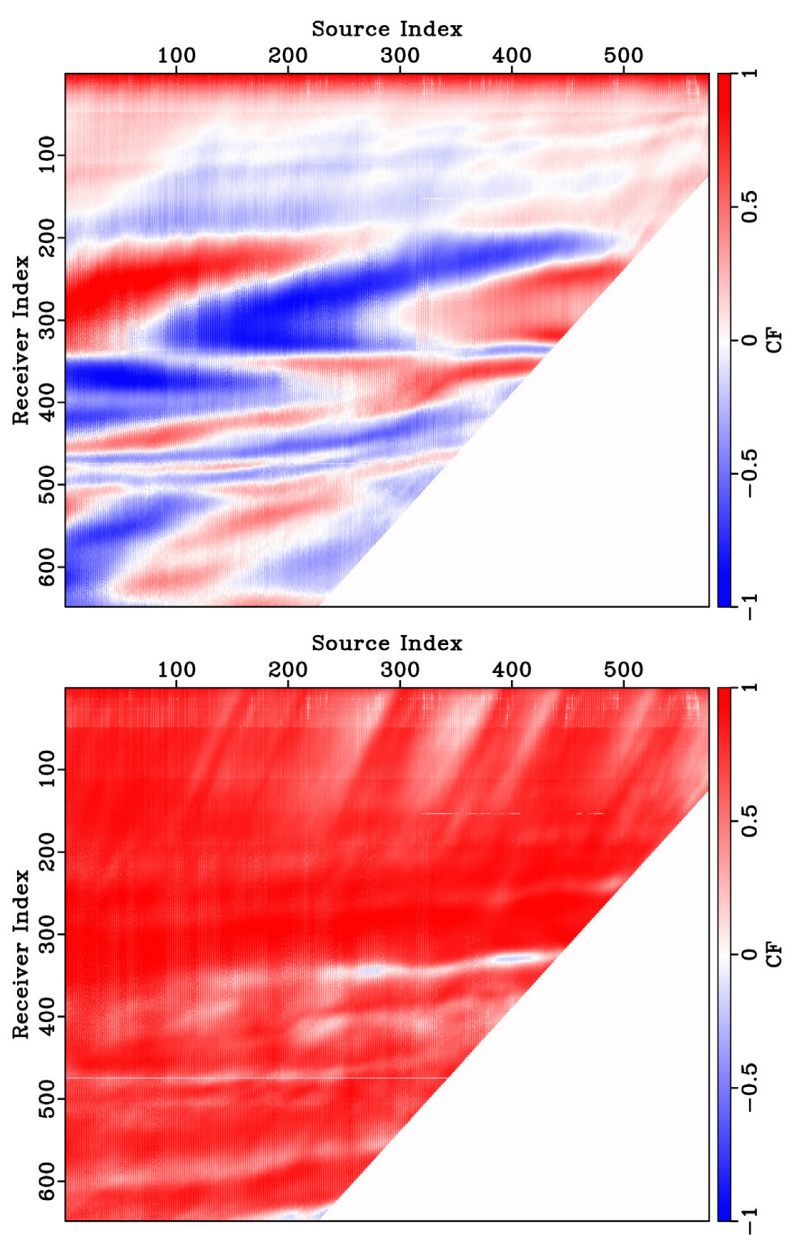

Figure 29. Correlation factor maps for all predicted traces using the (a) initial, (b) final velocity models. The zero values in the right corner of the maps are because of the unavailability of traces. Note that two identical signals produce a correlation factor of unity. requires to embrace the following changes:

$$
\begin{aligned}
\tilde{\mathbf{g}} & =-\left(\frac{\partial \tilde{\mathbf{L}}}{\partial \mathbf{m}} \tilde{\mathbf{u}}\right)^{T}\left(\tilde{\mathbf{L}}^{-1}\right)^{T} R^{T}\left(\mathbf{d}_{m o d}-\mathbf{d}_{o b s}\right) \\
& =2 \mathbf{m}_{b}<\nabla^{2} \tilde{\mathbf{u}}, \tilde{\mathbf{v}}>\quad \text { with } \quad \tilde{\mathbf{L}} \tilde{\mathbf{u}}=f \quad \& \quad \tilde{\mathbf{L}}^{T} \tilde{\mathbf{v}}=\mathbf{d}_{m o d}-\mathbf{d}_{o b s} .
\end{aligned}
$$

Therefore, the gradient evaluation of FWI, using FCT, is a simple pre-conditioner of the conventional formulation, due to the modification in the wave-equation operator, controlled by the parameter $\xi$. We propose to harness $\xi$, in descending order, to invert the fullbandwidth of the data set. We start the inversion with a large $\xi$ and relax it progressively to zero so that the final inverted model is achieved from conventional FWI. With large $\xi$ values, FCT attempts to retrieve the model's long-wavelength components from the data. With the maturity of the inversion process, FCT with smaller $\xi$ values starts recovering the fine-scale wavelengths. Some of the obvious features of our method are as follow:

(i) It can be easily extended to any objective functions, since required modifications take place only in the wave equation propagator, rather than the entire inversion setup.

(ii) A large $\xi$ promotes low frequencies in the wavefield, hence will produce a smooth gradient even from full bandwidth data. Unlike Gaussian kernel smoothing, it is a dynamic filter that tackles the convergence issues at stake.

(iii) It is more convenient than the popular multiscale approach presented by Bunks et al. (1995). In general, the signal-to-noise ratio in the low frequency bandwidth of seismic recordings is very poor, hence, it produces a noisy-gradient with the conventional approach. However, FCT produces a smooth gradient from full bandwidth data with a better signal-to-noise ratio. ${ }^{3}$ Finally, unlike conventional approaches, FCT does not require any preconditioning such as the use of band-pass filtering for the observed data.

(iv) It is very easy to implement within the existing FWI codes.

(v) At the end, it only requires a classical FWI $(\xi=0$, in this case) with a background model that has undergone multiple refinement over the FWI iterations for $\xi \neq 0$. Therefore, starting from a better initial model, we, finally, achieve the inverted model through classical FWI.

\subsection{Sensitivity kernel using FCT: from hollow to solid fat banana-kernel}

In seismic waveform tomography, the sensitivity kernel or the Fréchet derivative provides insights into the Fresnel zone, along the wavepath due to model perturbations (Woodward 1992; Tromp et al. 2005; van der Hilst \& de Hoop 2005; Djebbi et al. 2013). Therefore, it delineates the zone of model perturbations that contribute to the data residuals (difference between the predicted and the observed records). Thus, we, analyse the sensitivity kernels of a trace, with $3 \mathrm{~Hz}$ dominant frequency and for a velocity model, as shown in Fig. 5(a). Fig. 5(b) shows the model perturbations that yield non-zero differences in data residuals. As expected, the kernel for the seismic trace resembles a banana shape around the source and receiver positions (Figs $5 \mathrm{c}$ and $\mathrm{d}$ ). However, the kernel using FCT (Fig. 5d), is slightly fatter than with the conventional method (Fig. 5c).

Next, we carry out the same experiment with $10 \mathrm{~Hz}$ dominant frequency. As expected, the conventional sensitivity kernel as shown in Fig. 6(a) incurs a wider insensitive zone across the geometrical

\footnotetext{
${ }^{3}$ Details are provided in Section 2.4 .
} 

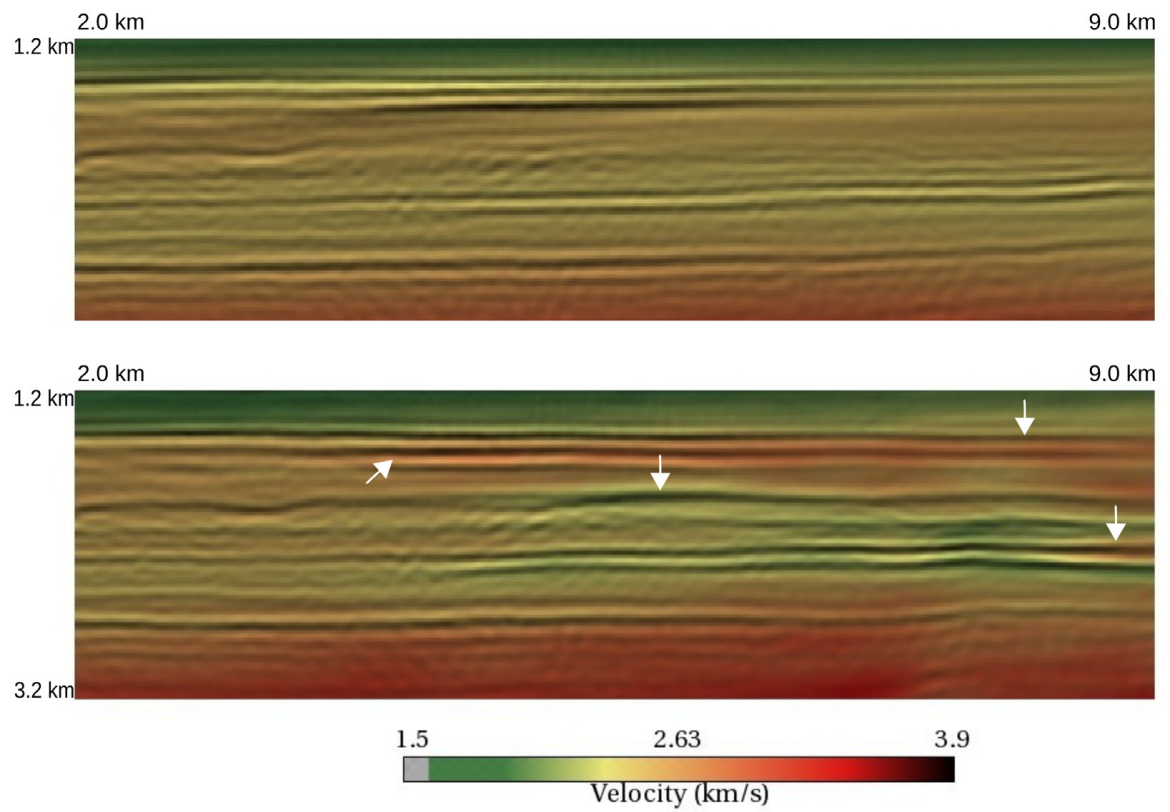

Figure 30. Reverse-time-migration images overlaying over the initial (top panel) and final (bottom panel) migration velocity models. As indicated by the arrows, final velocity model better delineates the reflectors than the starting model.
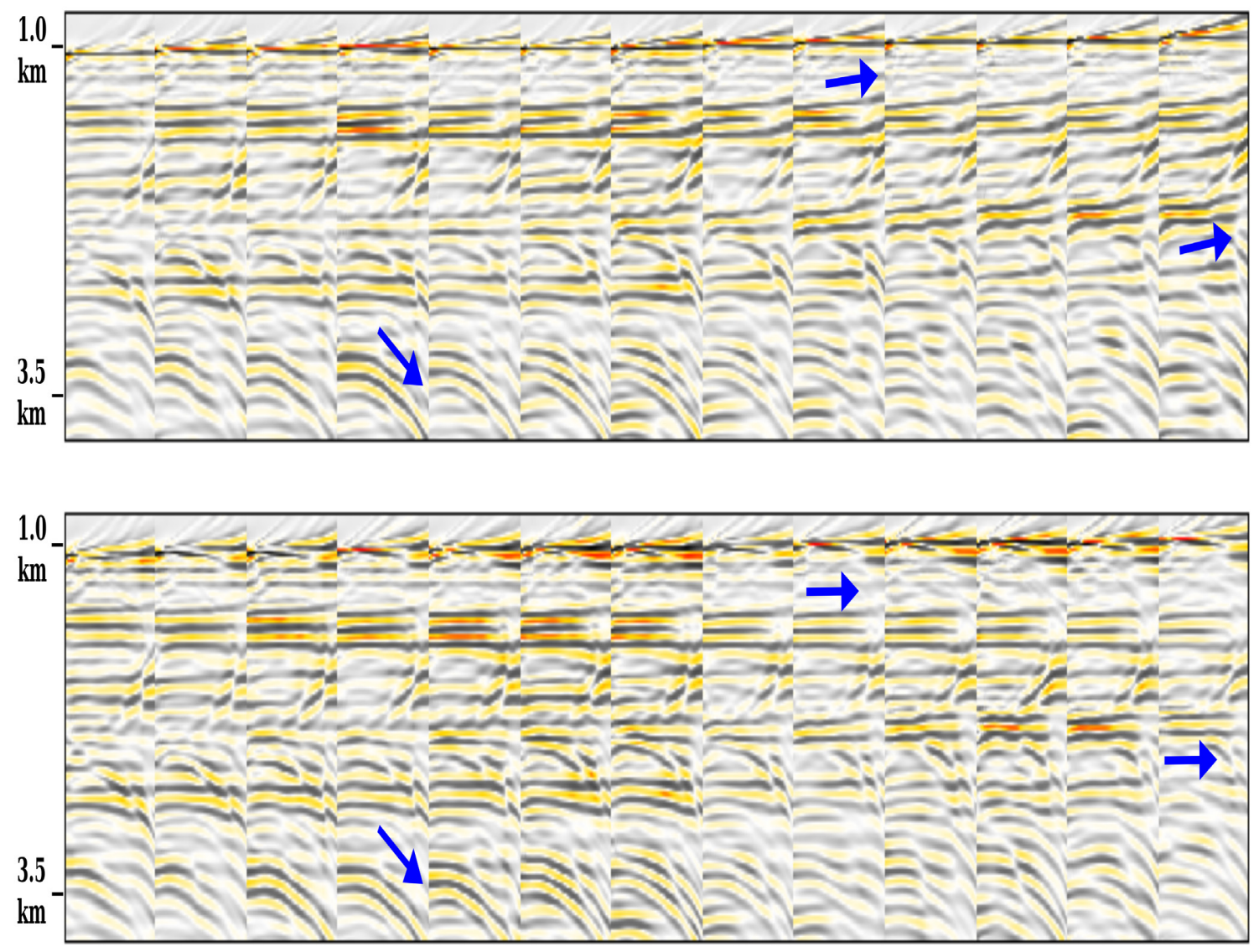

Figure 31. ADCIGs below the see bottom of initial (top panel) and final inverted (bottom panel) models, computed at an interval of $0.4 \mathrm{~km}$ in the region of $x=[3.0 \mathrm{~km}, 8.2 \mathrm{~km}]$. The arrows indicate general trends of events in ADCIGs. Compared to the top panel, the events are flatter in the bottom panel which signify improvements in the retrieval process of velocity field from sesimic data using FCT approach. 


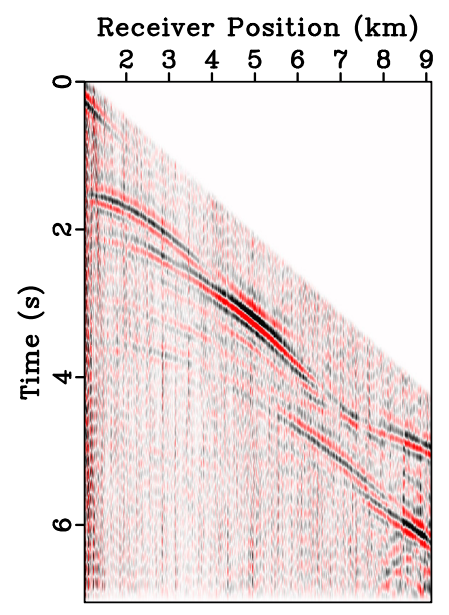

(a)

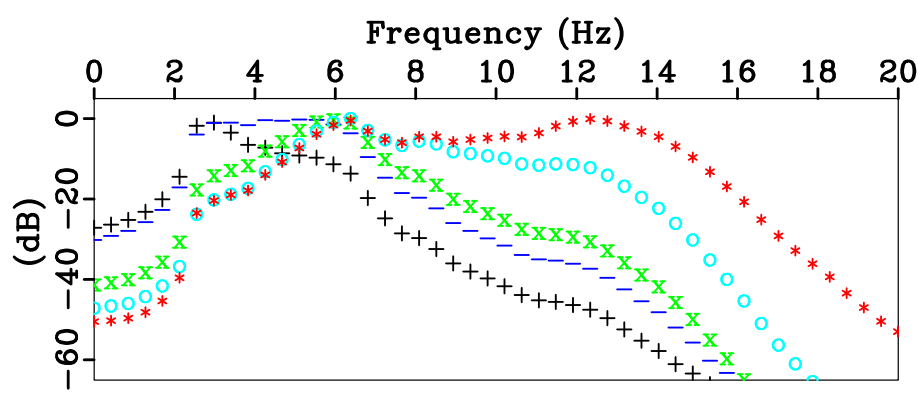

(b)

Figure 32. (a) A shot gather for the first stage of multiscale FWI method, (b) its average power spectrum (normalized) shown by the marker symbol ' + '. The other symbols '-', ' $x$ ', 'o' and '*' represent the frequency content of the shot-gather used in different stages of the conventional multiscale method.

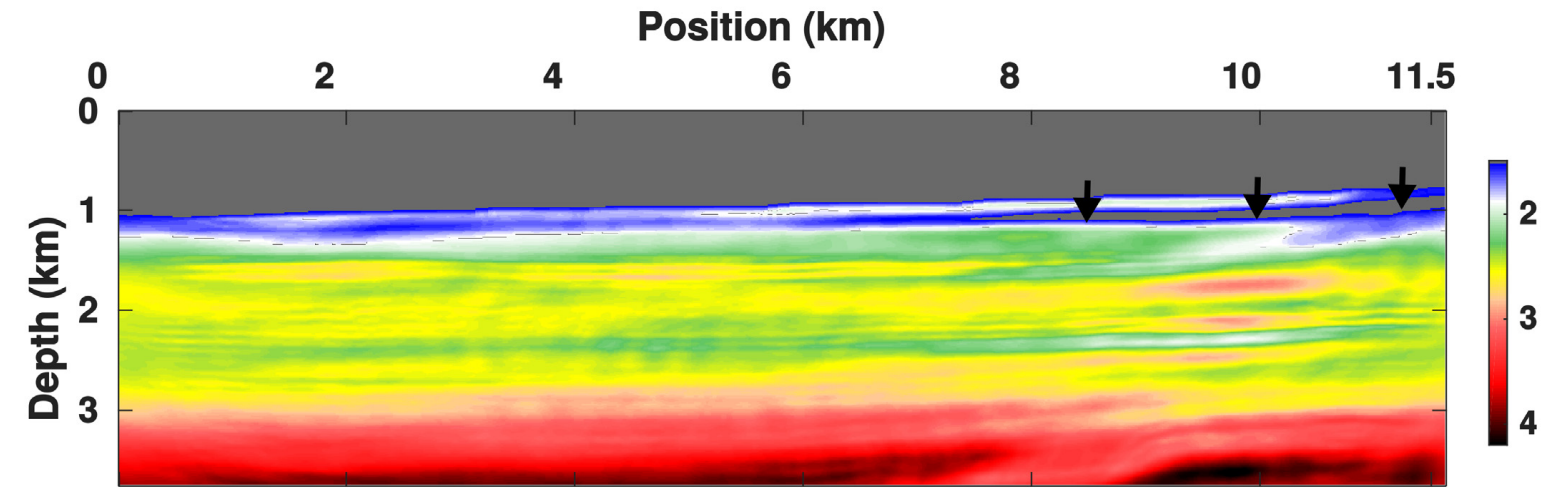

Figure 33. Final inverted model using conventional FWI (Bunks et al. 1995). The arrows point to a zone with water layer velocity which is most-probably an artifact of FWI.

ray path in comparison with the previous case. However, FCT has a profound impact on reducing that region of zero sensitivity (Fig. 6b). Moreover, it enjoys a kernel response similar to that of $3 \mathrm{~Hz}$ data (Fig. 5).

\subsection{Implementation strategy of FWI using FCT}

The essence of our proposed method is that it is very easy to implement in the existing framework of FWI. It requires no changes besides the modifications in the modelling engine as shown in Algorithm 1. Computational costs due to the modifications are negligible. It is worth mentioning that the exclusion of the non-linear step from original FCT has reduced the bulk of floating point operations such as finding maxima, minima, etc., thus, the computational time. Given the flux-corrected wavefield, the gradient computation is very straightforward and only requires a temporal cross-correlation process between the source wavefield and the back-propagated adjoint wavefield. In doing so, we usually need to store the entire source wavefield, often referred to as history saving scheme, or include an extra extrapolation step to propagate the source wavefield from its storage to the boundary (Berkhout 1988; Clapp 2008), often referred as boundary saving scheme. With advancements in acquisition process, such as multicomponent and wide azimuth, which are cultivating huge data sets of the order of terabytes in size, the boundary saving scheme is more attractive albeit we will incur an additional wavefield extrapolation compared to the history saving scheme. However, it is possible to use the history saving scheme for the examples being considered here. Both schemes to compute the gradient directions using FCT are reported in Algorithms 2 and 3. Unlike conventional FWI, the history-saving scheme in FCT requires an additional wavefield extrapolation to predict seismic records in order to prepare the adjoint source, as shown in Algorithm 3. However, number of wavefield extrapolation remains same for both the conventional and FCT-based FWI, using boundary saving scheme.

\section{EXAMPLES}

\subsection{Marmousi II}

We demonstrate an application of the proposed method to the Marmousi II velocity model. There are several normal faults and resulting tilted blocks towards the centre, as well as complicated fine structures particularly towards the right, and horizontal layering towards the left side of the model. We display the true synthetic 

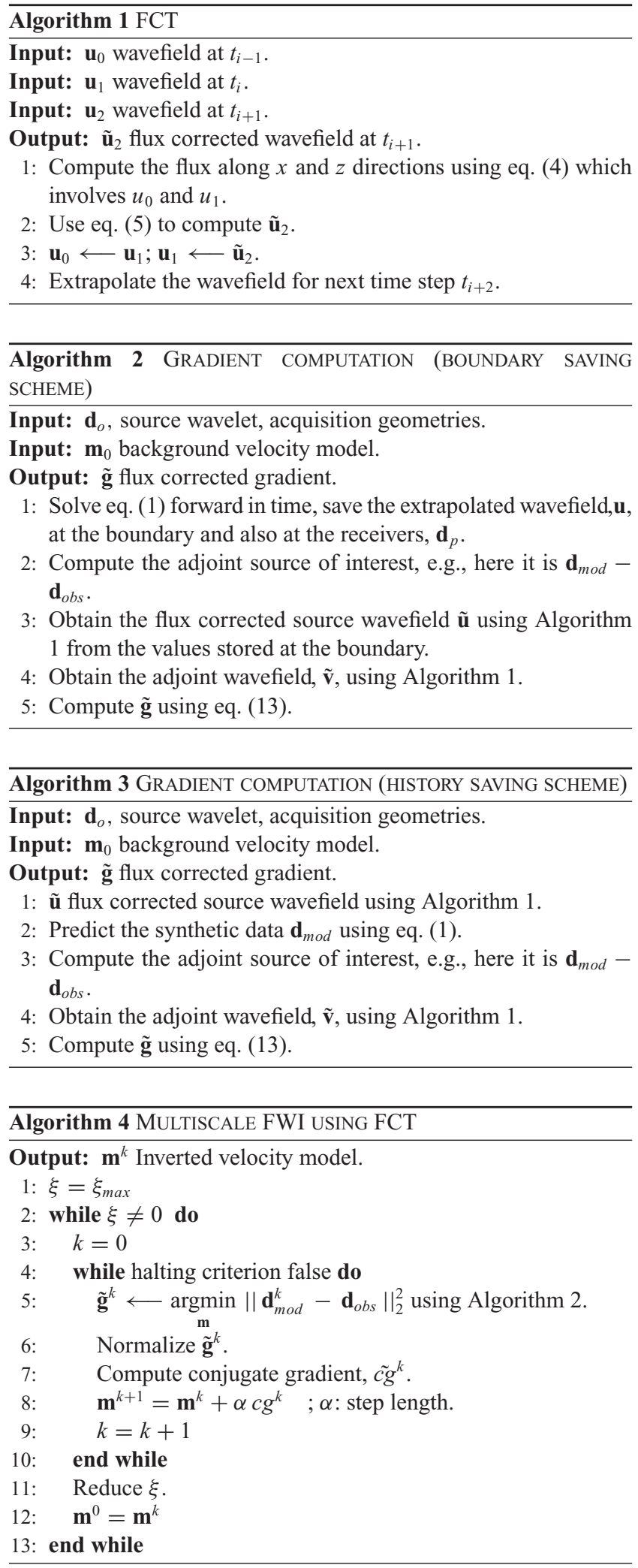

model of our consideration in Fig. 1. The model contains 679 and 139 gridpoints along the $x$ and $z$ directions, respectively, at an equal interval of $0.025 \mathrm{~km}$. There are 300 sources placed between $x=$ 0.5 and $15.45 \mathrm{~km}$ at intervals of $0.05 \mathrm{~km}$, and a depth of $z=$ $0.025 \mathrm{~km}$. Data are recorded by a $10.0-\mathrm{km}$-long streamer, starting from $0.2 \mathrm{~km}$ at the near offset for 8 seconds. We set the length of the streamer to vary with the position of the sources in order to emulate the typical marine acquisition geometry. Fig. 7 confirms the poor acquisition geometry towards the right part of the model. The streamer is floated at the same depth as the source-line. A Ricker wavelet of $10 \mathrm{~Hz}$ dominant frequency filtered by a high-cut filter at $13 \mathrm{~Hz}$, is used to synthesize the observed data with the free surface boundary condition. Fig. 8 displays the source wavelet and its power spectrum. Next, we show two shot gathers from sources placed at $x=6$ and $14 \mathrm{~km}$ in Figs 9(a) and (b), respectively. We display the frequency content of the shot gather from Fig. 9(a) in Fig. 10.

We start the inversion process with a model (Fig. 11), in which the velocity increases linearly with depth from the water bottom. Fig. 12(a) shows the gradient direction computed at the first iteration of FWI using $\xi=0.20$. As expected, it accentuates the smooth features of the true model that are missing from the initial model (Fig. 12b). As we decrease $\xi$ over the iterations, FWI progressively attempts to capture the fine-scale features of the model (Fig. 13). The inversion starts by with the recovery of the smooth tomographic part, especially at the shallow region, then progressively propagates further down with an enhancement in the resolution of the model. We display the final inverted model in Fig. 14 which depicts a close resemblance with the true model shown in Fig. 1.

We analyse the evolution of predicted data over the FWI iterations shown in Fig. 15. For comparison purposes, we interleave observed and predicted seismic records from the source at $x=6 \mathrm{~km}$. The initial velocity model (Fig. 11) is very smooth and hence produces no reflection events apart from the water bottom represented in Fig. 15(a). Also, we observe that the diving waves produced in the initial model, arrive at the receivers, especially at far-offset, more than a cycle later than those in observed recordings. FWI after the first iteration attempts to minimize that delay in arrivals as shown in Fig. 15(b). However, the velocity model, shown in Fig. 12(b), is not mature enough to produce any reflection events that match the observed counterparts, especially in the near-offset recordings. The velocity model, shown in Fig. 13(a), generates seismic records that start resembling the observed data. For instance events pointed out by ellipses in Fig. 15(c) suggests clear improvements in the velocity model. At the 51st iteration, we retrieve a velocity model (Fig. 13(b) that depicts a smoother version of the true model, and hence, a better matching of the waveform (Fig. 15d). The final velocity model, Fig. 14, produces a data set that is almost identical to the observed ones as shown in Fig. 15(e). Fig. 16 shows the percentage of errors ${ }^{4}$ for the initial and the final velocity models. Taking the innate limitations such as the finite frequency bandwidth data set, incomplete acquisition aperture and so forth into account, Fig. 14 suggests that our proposed method successfully retrieves most, if not all of the true velocity features. Fig. 17 shows the history of the $L^{2}$ norm of data-residual. Next, we show the failure that conventional FWI experiences for the full-bandwidth data in Fig. 18(a). However, the multiscale method (Bunks et al. 1995) recovers the model as shown in Fig. 18(b).

\subsection{Marmousi II: noisy data}

We demonstrate the versatility of FCT-based FWI on a noisy-data set from the Marmousi II velocity model. In order to prepare the observed data as shown in Fig. 19(a), we add white-noise to the data set of the previous example (Fig. 9a). The SNR at the lower frequency bandwidths of the data is very poor, that is negative in

${ }^{4}$ per cent error $=100\left|1-\frac{\mathbf{m}}{\mathbf{m}_{\text {true }}}\right|$. 
$\mathrm{dB}$ scale. For example, the SNR for the frequency bandwidth of \{ $0-1 \mathrm{~Hz}, 0-2 \mathrm{~Hz}, 0-3 \mathrm{~Hz}, 0-4 \mathrm{~Hz}, 0-5 \mathrm{~Hz}, 0-6 \mathrm{~Hz}\}$ are $\{-42 \mathrm{~dB}$, $-24 \mathrm{~dB},-12 \mathrm{~dB},-4 \mathrm{~dB},-1 \mathrm{~dB}, 5 \mathrm{~dB}\}$, respectively. We compare the power spectra of noisy and noise-free data in Fig. 9(b) which shows a clear dominance of noise over signal in the lower frequency bandwidth (below than $5 \mathrm{~Hz}$ ).

We start the inversion process with a linear $v(z)$ model as shown in Fig. 11. We show the gradient direction computed at the first iteration of FWI using $\xi=0.15$ in Fig. 20(a). ${ }^{5}$ Fig. 20(b) shows the final inverted velocity model using our proposed method. The conventional multiscale FWI severely fails to recover the model as shown in Fig. 21. The computed gradient even at the first iteration (Fig. 21a) is very noisy, and also contaminated with short-scale wavelengths (migration components) rather than it being smooth (tomographic components) due to the poor SNR of the lower frequency bandwidths of the observed recordings. As a consequence, conventional FWI retrieved a model (Fig. 21b) which is very far fromthe true global minimum model (Fig. 1). On the other hand, FCT enjoys a much better convergence than classical multiscale FWI, even in the presence of noise in the observed data.

Next, we increase the noise level further to investigate the breakdown of FCT in presence of extreme noise. Fig. 21 shows a shot gather and its power spectrum. The comparison of power spectrum of the shot gather with those from the last two examples confirms the hike in the noise level. For example, the SNR for the bandwidths of $\{0-1 \mathrm{~Hz}, 0-2 \mathrm{~Hz}, 0-3 \mathrm{~Hz}, 0-4 \mathrm{~Hz}, 0-5 \mathrm{~Hz}, 0-6 \mathrm{~Hz}, 0-7 \mathrm{~Hz}\}$ is $\{-51 \mathrm{~dB},-32 \mathrm{~dB},-24 \mathrm{~dB},-12 \mathrm{~dB},-7 \mathrm{~dB},-2 \mathrm{~dB}, 1 \mathrm{~dB}\}$, respectively. As expected, conventional FWI fails to retrieve the model as shown in Fig. 23(a). We show the final inverted model using our porposed method in Fig. 23(b). Although the left part of the model is contaminated with the artifacts due to the noise present in the recorded seismic signal, we manage to retrieve the right part, especially the shallow region. Nevertheless. FCT-based FWI reconstructs the inverted model way better than the conventional multiscale scheme.

\subsection{Field data set}

This example corresponds to a 2-D field marine data set acquired in North-Western Australia Continental shelf using a Broadseis system with a variable depth streamer. This type of acquisition technique enriches the low frequency content of the data set (Soubaras \& Dowle 2010). The minimum available frequency of the data set is around $3.0 \mathrm{~Hz}$. We consider a model of $11.6 \mathrm{~km}$ long and $3.75 \mathrm{~km}$ deep in size. Accordingly, only 576 out of 1824 shot-gathers with a source interval of approximately $0.01875 \mathrm{~km}$ are selected for inversion purposes. The length of the streamer is $8.3 \mathrm{~km}$ : it contains 648 receivers with the near offset at $0.169 \mathrm{~km}$. Fig. 24 represents the number of receivers per shot fit inside the area of investigation. It confirms the poor acquisition sampling towards the right side of the model. We display two shot gathers from the sources placed at $x=1.0$ and $9.0 \mathrm{~km}$ in Fig. 25. Fig. 26 shows the frequency content of the shot displayed in Fig. 25(a).

We choose to minimize the normalized cross-correlation objective function (Routh et al. 2011; Choi \& Alkhalifah 2012) instead of the $L^{2}$ norm of the data misfit. Borrowing the inverted wavelet from Kalita \& Alkhalifah (2017), we start the inversion with a model (Fig. 27a), derived from the given brute-stack time-domain

\footnotetext{
${ }^{5}$ Although smooth, the resulting update direction using $\xi=0.20$ cannot reduce the data misfit. Hence, FCT-based FWI automatically considers the next available $\xi$ value which is 0.15 in this case.
}

velocity information. Additionally, we keep the water layer velocity unchanged throughout the inversion process. Fig. 27 shows the evolution of the velocity model. As expected, the inversion starts recovering the low wavenumber, and then the high wavenumbers. We retrieve the final velocity model as shown in Fig. 27(d), featuring many sharp contrasting regions, especially at depths of $z=1.5$ and $3.0 \mathrm{~km}$.

We validate the inversion result by the following QC measures:

(i) We compare the observed data with synthetic counterparts that computed by applying acoustic constant density wave equation into the model. We interleave synthetic and observed shot records in Fig. 28 to accentuate the matching the kinematics of associated arrivals. Fig. 28(a) shows the matching of data from the initial model and Fig. 28(b) from the final model. The coherency in the events that are shown in Fig. 28(b) confirms improvements in the final velocity model (Fig. 27d), compared to the initial model (Fig. 27a).

(ii) To make the data matching comparison more quantitative, we display the correlation factor (CF), defined as (Wu \& Alkhalifah 2015)

$$
C F=\frac{<\mathbf{d}_{m o d}, \mathbf{d}_{o b s}>}{\sqrt{<\mathbf{d}_{m o d}, \mathbf{d}_{m o d}><\mathbf{d}_{o b s}, \mathbf{d}_{o b s}>}} .
$$

Two identical signals yield a CF of unity. We compute the CF for each pair of traces in Fig. 29. The zero values in the right corner of the maps, is due to the unavailability of traces (Fig. 24). CF's map confirms the better matching of synthetic data with the observed counterparts for the final velocity model than the initial model.

(iii) We observe improvements in the migration image also. The final velocity model accentuates the reflectors much better than the initial does (Fig. 30).

(iv) For velocity analysis, it is often useful to display partial image profiles as a function of reflection angles, known as angle domain common image gathers (ADCIGs). The erroneous velocities produce non-flat events in ADCIGs. We have computed ADCIGs (Fig. 31) using excitation method (Kalita \& Alkhalifah 2016) ${ }^{6}$ for initial (Fig. 27a) and final inverted (Fig. 27d) models at every interval of $0.4 \mathrm{~km}$, starting from 3.0 to $8.2 \mathrm{~km}$. It shows some of the events, as pointed out by the arrows, are flatter with the inverted model compared to initial model.

In order to compare the outcomes of FCT method with conventional FWI, we perform the multiscale approach (Bunks et al. 1995) on this seismic data set, starting the inversion at the lower frequency bandwidth and progressively including the higher frequencies. We display a shot-gather and its frequency content that is involved in the first-stage of the multiscale scheme in Fig. 32. In addition, we overlay all the frequency bandwidths of consideration for various stages of the inversion in Fig. 32(b). Next, we display the inversion result in Fig. 33. We retrieve a thin layer of water velocity at $x>8.0 \mathrm{~km}$ below the sea-bottom, as pointed out by the arrows, which is probably one of the artifacts of FWI incurred because of cycle-skipping. An enlarged region from Fig. 33 as shown in Fig. 34 suggests that the velocity model obtained using FCT method (Fig. 34a) contains more prominent and continuous geological features than that using conventional FWI (Fig. 34b). We validate this claim by comparing ADCIGs in the region of consideration (Fig. 35). Lastly, we produce the RTM image in Fig. 36 using the migration velocity model

${ }^{6}$ Excitation approach reduces computational overburden of ADCIGs' evaluation. 


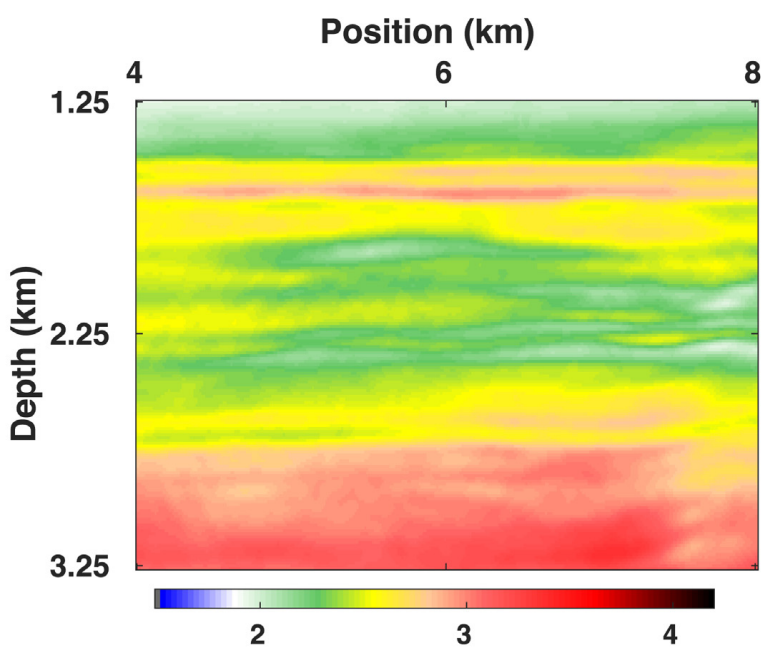

(a)

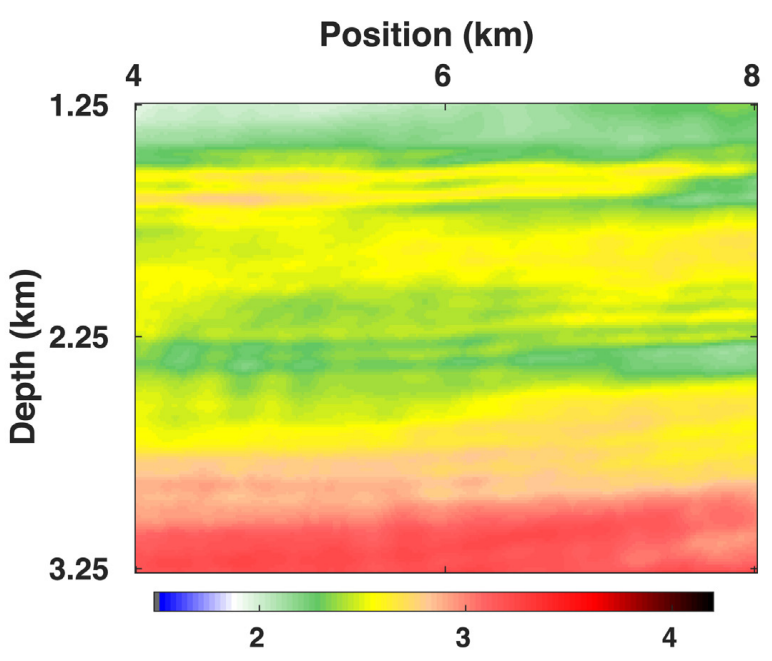

(b)

Figure 34. Enlarged section of final inverted models using (a) FCT and (b) conventional method. It suggests that conventional FWI failed to retrieve some of the high and low velocity zones as recovered by the FCT method.
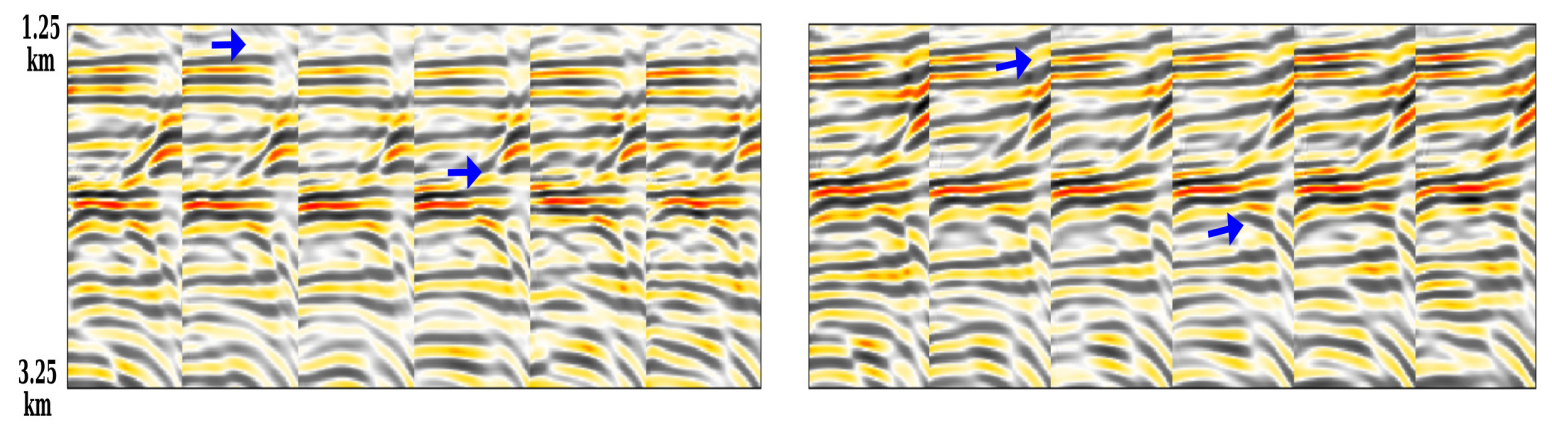

Figure 35. ADCIGs computed at $x=\{7.0,7.2,7.4,7.6,7.8,8.0\} \mathrm{km}$ for a region of $z=1.25 \mathrm{~km}$ to $z=3.25 \mathrm{~km}$ using velocity models obtained by FCT approach (left-hand panel) and conventional method (right-hand panel). The arrows indicate a general trend of the events, which are, in general, flatter in the left-hand panel compared to the right-hand panel. It suggests that our approach retrieved velocity parameters better than conventional multiscale method from the field data set of consideration.

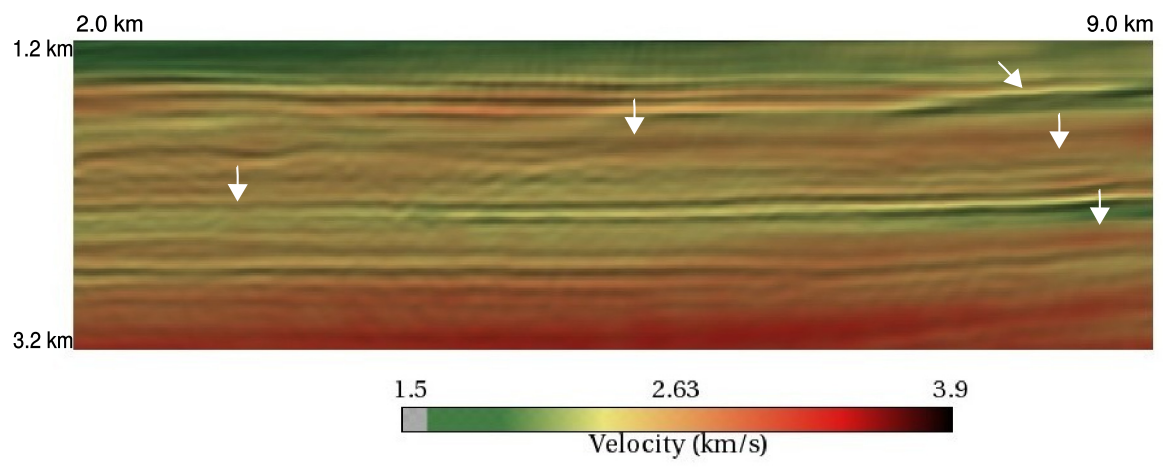

Figure 36. Enlarged section of the reverse-time-migration image overlaying over the final migration velocity model obtained using conventional multiscale FWI. The reflectors as pointed out the by the arrows are less focused, flat and continuous compared to the FCT counterparts in Fig. 30 (bottom panel).

obtained by conventional multiscale method. Comparing it with the RTM image in Fig. 30(bottom panel), we observe that the events are better focused, more continuous and flatter with the inverted migration velocity of FCT method.

\section{DISCUSSION}

Because of the exclusion of the anti-diffusion step from the original version of Fei \& Larner (1995), the FCT operator becomes linear, and hence, it can easily be employed in an adjoint tomography. It 
promotes low frequencies in the wavefield, thus the smooth gradient from a high-frequency dominant source. It is worth mentioning that, unlike many other recent algorithms, it requires no tampering with the objective function of FWI to produce a smooth gradient direction. Neither we do require cumbersome efforts to adopt the algorithm into the existing FWI code. However, it is not a panacea to resolve the endemic cycle skipping in FWI once and for all, especially in absence of adequate low-frequencies in the data set. For instance, it failed to retrieve the velocity field starting from a $v(z)$ model in the case of the field data example.

The predicted seismic gathers using FCT, especially for large $\xi$ values are smoother than the full-bandwidth observed data. Hence, they cannot be considered for the misfit measurements. Therefore, we require a conventional modeling step $(\xi=0)$ in order to compute synthetic data for the preparation of adjoint sources of FWI. As a consequence, we require an additional wavefield extrapolation in case we opt the history saving scheme for inversion machinery ${ }^{7}$ However, as $\xi$ approaches to zero, we can safely consider the predicted seismic data using FCT to the inversion scheme. For $\xi=0$, it is nothing but a conventional FWI.

The smoothing parameter, $\xi$, controls the success and convergence rate of FCT algorithm in FWI. As pointed out by Boris \& Book (1973), $\xi$ depends on the numerical FD scheme under consideration, sampling intervals and velocity model. In their work, Boris $\&$ Book (1973) set $\xi=0.125$ in order to remove the undesired ripples of a shock profile. Fei \& Larner (1995) considered $\xi$ to be less than or equal to 1 . As a rule of thumb, $\xi$ should not be too large to incur the numerical stability of wave equation. We heuristically select plausible $\xi=\xi_{\max }$ and gradually decrease $\xi$ to zero in the proposed multiscale inversion scheme. ${ }^{8}$ More research will be necessary to optimize the selection procedure of $\xi$ in order to reduce the redundancy.

The main goal of this study was to develop a robust and easy-toimplement strategy in waveform inversion to mitigate the problem of cycle-skipping. We have done so by developing FCT for a 2-D acoustic medium with a constant density. However, our premise of a simplistic physical behavior of the wavefield may not be valid for a complicated earth model. Therefore, we cannot rule out the challenges that arise when inverting more complex provinces, using FCT. Thanks to the previous work of Fei \& Larner (1995) and Zheng et al. (2006), the work presented here may also be extended to a non-linear 3-D elastic medium with an anisotropic behavior. Further studies on more complicated media will shed some light on the versatility of our method, and, will be reported in future papers.

\section{CONCLUSIONS}

We developed a novel multiscale approach in FWI using flux correction transport, which serves as an improved alternative to the conventional hierarchical frequency scaling approach. It involves a parameter $\xi$, which governs the smoothness of the wavefield, hence, the gradient. FCT with a large $\xi$ promotes available low frequencies, hence a smooth gradient from the high-frequency dominant seismic data. A gradual decrease of $\xi$ to zero results in conventional

\footnotetext{
${ }^{7}$ For large 3-D scale problems, FWI prefers boundary saving scheme. This scheme for FCT-based FWI requires same number of modelling steps with that for conventional FWI.

${ }^{8}$ In order to select plausible $\xi_{\text {max }}$ under the in situ experimental conditions, we do forward modelling for the initial velocity model using a set of $\xi$ values just before initiating FWI.
}

FWI for the full bandwidth of the data set. Besides its robustness, this algorithm can easily be adapted to the existing FWI code. The modifications in FCT are very cheap, requiring only a few additional algebraic operations to the wavefield solution. We validated the proposed method on data sets of Marmousi II model. FCTbased FWI successfully retrieved most of the model. Moreover, we demonstrated it could be applied to a field data set in which the FCT method clearly outperforms the conventional multiscale FWI method.

\section{ACKNOWLEDGEMENTS}

The research reported in this publication was supported by funding from King Abdullah University of Science and Technology (KAUST). We would like to thank KAUST for its support, as well as, all members of the Seismic Wave Analysis Group (SWAG) for the fruitful discussions. We also thank Dr. David Ketcheson from Applied Mathematics and Computational Science at KAUST for his valuable suggestions. For computer time, this research used the resources of the Supercomputing Laboratory and IT Research Computing at KAUST. In addition, this research used the resources of the Core Labs of KAUST. We acknowledge Thomas Theussl from Visualization Core Laboratory at KAUST, for his assistance in displaying some of the results reported in this work. The real data shown in this study are proprietary and provided by courtesy of CGG.

\section{REFERENCES}

Alkhalifah, T., 2015. Scattering-angle based filtering of the waveform inversion gradients, Geophys. J. Int., 200, 363-373.

Alkhalifah, T. \& Choi, Y., 2016. A source of low frequencies-smoothing the wavefield, in Proceedings of the 78th EAGE Conference and Exhibition 2016, Vienna.

Alkhalifah, T. \& Wu, Z., 2016. The natural combination of full and imagebased waveform inversion, Geophys. Prospect., 64(1), 19-30.

Allemand, T. \& Lambaré, G., 2015. Combining full waveform inversion and tomography: full waveform inversion-guided tomography, in Proceedings of the 77th EAGE Conference and Exhibition 2015, Madrid, Spain.

Berkhout, A., 2014. An outlook on the future of seismic imaging, part III: Joint migration inversion, Geophys. Prospect., 62(5), 950-971.

Berkhout, A.J., 1988. A holographic approach to acoustic control, J. Audio Eng. Soc., 36(12), 977-995.

Bharadwaj, P., Mulder, W. \& Drijkoningen, G., 2016. Full waveform inversion with an auxiliary bump functional, Geophys. J. Int., 206(2), 10761092.

Biondi, B. \& Almomin, A., 2014. Simultaneous inversion of full data bandwidth by tomographic full-waveform inversion, Geophysics, 79(3), WA129-WA140.

Book, D.L., Boris, J.P. \& Hain, K., 1975. Flux-corrected transport II: generalizations of the method, J. Comput. Phys., 18(3), 248-283.

Boonyasiriwat, C., Valasek, P., Routh, P., Cao, W., Schust er, G.T. \& Macy, B., 2009. An efficient multiscale method for time-domain waveform tomography, Geophysics, 74(6), WCC59-WCC68.

Boris, J.P. \& Book, D., 1976. Flux-corrected transport. III. Minimal-error fct algorithms, J. Comput. Phys., 20(4), 397-431.

Boris, J.P. \& Book, D.L., 1973. Flux-corrected transport. I. Shasta, a fluid transport algorithm that works, J. Comput. Phys., 11(1), 38-69.

Boris, J.P. \& Book, D.L., 1997. Flux-corrected transport, J. Comput. Phys., 135(2), 172-186.

Bozdağ, E., Trampert, J. \& Tromp, J., 2011. Misfit functions for full waveform inversion based on instantaneous phase and envelope measurements, Geophys. J. Int., 185(2), 845-870.

Bunks, C., Saleck, F.M., Zaleski, S. \& Chavent, G., 1995. Multiscale seismic waveform inversion, Geophysics, 60(5), 1457-1473. 
Choi, Y. \& Alkhalifah, T., 2012. Application of multi-source waveform inversion to marine streamer data using the global correlation norm, Geophys. Prospect., 60(4), 748-758.

Choi, Y. \& Alkhalifah, T., 2018. Time-domain full-waveform inversion of exponentially damped wavefield using the deconvolution-based objective function, Geophysics, 83(2), R77-R88.

Clapp, R.G., 2008. Reverse time migration: saving the boundaries, Stanford Exploration Project, p. 136.

Djebbi, R. et al., 2013. Finite frequency traveltime sensitivity kernels for acoustic anisotropic media: angle dependent bananas, in Proceedings of the 2013 SEG Annual Meeting, Society of Exploration Geophysicists, Houston, Texas, the USA.

Engquist, B. \& Froese, B.D. 2013. Application of the wasserstein metric to seismic signals, arXiv preprint arXiv:1311.4581.

Fei, T., 1994. Elimination of numerical dispersion in finite-difference modeling and migration by flux-corrected transport, Colorado-School of Mines.

Fei, T. \& Larner, K., 1995. Elimination of numerical dispersion in finite-difference modeling and migration by flux-corrected transport, Geophysics, 60(6), 1830-1842.

Kalita, M. \& Alkhalifah, T., 2016. Common-image gathers using the excitation amplitude imaging condition, Geophysics, 81(4), S261-S269.

Kalita, M. \& Alkhalifah, T., 2017. Efficient full waveform inversion using the excitation representation of the source wavefield, Geophys. J. Int., 210, 1581-1594.

Kazei, V., Tessmer, E. \& Alkhalifah, T., 2016. Scattering angle-based filtering via extension in velocity, in Proceedings of the SEG Technical Program Expanded Abstracts 2016, pp. 1157-1162, Society of Exploration Geophysicists, Dallas, Texas USA.

Lailly, P. 1983. The seismic inverse problem as a sequence of before stack migrations, in Conference on Inverse Scattering: Theory and Application, pp. 206-220, Society for Industrial and Applied Mathematics.

Löhner, R., Morgan, K., Peraire, J. \& Vahdati, M., 1987. Finite element flux-corrected transport (FEM-FCT) for the euler and Navier-Stokes equations, Int. J. Numer. Methods Fluids, 7(10), 1093-1109.

Luo, S., Sava, P. et al., 2011. A deconvolution-based objective function for wave-equation inversion, in Proceedings of the 2011 SEG Annual Meeting, Society of Exploration Geophysicists, San Antonio, Texas, USA.

Métivier, L., Brossier, R., Mérigot, Q., Oudet, E. \& Virieux, J., 2016. Measuring the misfit between seismograms using an optimal transport distance: application to full waveform inversion, Geophys. Suppl. Mon. Not. R. Astron. Soc., 205(1), 345-377.

Mora, P., 1989. Inversion= migration+ tomography, Geophysics, 54(12), $1575-1586$.

Oh, J.-W. \& Alkhalifah, T., 2018. Full waveform inversion using envelopebased global correlation norm, Geophys. J. Int., 213(2), 815-823.

Operto, S., Virieux, J., Dessa, J.-X. \& Pascal, G., 2006. Crustal seismic imaging from multifold ocean bottom seismometer data by frequency domain full waveform tomography: application to the eastern nankai trough, J. geophys. Res.: Solid Earth, 111(B9), doi:10.1029/2005JB003835.

Plessix, R.-E., 2006. A review of the adjoint-state method for computing the gradient of a functional with geophysical applications, Geophys. J. Int., 167(2), 495-503.

Pratt, R.G., 1990. Inverse theory applied to multi-source cross-hole tomography, Geophys. Prospect., 38(3), 311-329.

Pratt, R.G., Shin, C. \& Hick, G., 1998. Gauss-Newton and full Newton methods in frequency-space seismic waveform inversion, Geophys. J. Int., 133(2), 341-362.
Ravaut, C., Operto, S., Improta, L., Virieux, J., Herrero, A. \& Dell'Aversana, P., 2004. Multiscale imaging of complex structures from multifold wide-aperture seismic data by frequency-domain full-waveform tomography: application to a thrust belt, Geophys. J. Int., 159(3), 1032-1056.

Routh, P. et al., 2011. Encoded simultaneous source full-wavefield inversion for spectrally shaped marine streamer data, in Proceedigns of the SEG Technical Program Expanded Abstracts 2011, pp. 2433-2438, Society of Exploration Geophysicists, San Antonio, Texas, USA.

Sirgue, L. et al., 2003. Inversion de la forme d'onde dans le domaine fréquentiel de données sismiques grands offsets, PhD thesis, Paris 11.

Sirgue, L. \& Pratt, R.G., 2004. Efficient waveform inversion and imaging: a strategy for selecting temporal frequencies, Geophysics, 69(1), 231-248.

Soubaras, R. \& Dowle, R., 2010. Variable-depth streamer-a broadband marine solution, First Break, 28(12).

Symes, W.W., 2008. Migration velocity analysis and waveform inversion, Geophys. Prospect., 56(6), 765-790.

Tarantola, A., 1984. Inversion of seismic reflection data in the acoustic approximation, Geophysics, 49(8), 1259-1266.

Tóth, G. \& Odstrčil, D., 1996. Comparison of some flux corrected transport and total variation diminishing numerical schemes for hydrodynamic and magnetohydrodynamic problems, J. Comput. Phys., 128(1), 82-100.

Tromp, J., Tape, C. \& Liu, Q., 2005. Seismic tomography, adjoint methods, time reversal and banana-doughnut kernels, Geophys. J. Int., 160(1), 195216.

van der Hilst, R.D. \& de Hoop, M.V., 2005. Banana-doughnut kernels and mantle tomography, Geophys. J. Int., 163(3), 956-961.

Van Leeuwen, T. \& Mulder, W.A., 2010. A correlation-based misfit criterion for wave-equation traveltime tomography, Geophys. J. Int., 182(3), 13831394.

Virieux, J. \& Operto, S., 2009. An overview of full-waveform inversion in exploration geophysics, Geophysics, 74(6), WCC1-WCC26.

Warner, M. \& Guasch, L., 2016. Adaptive waveform inversion: theory, Geophysics, 81(6), R429-R445.

Woodward, M.J., 1992. Wave-equation tomography, Geophysics, 57(1), 1526

Wu, R.-S., Luo, J. \& Wu, B., 2014. Seismic envelope inversion and modulation signal model, Geophysics, 79(3), WA13-WA24.

Wu, Z. \& Alkhalifah, T., 2015. Simultaneous inversion of the background velocity and the perturbation in full-waveform inversion, Geophysics, 80(6), R317-R329.

Wu, Z. \& Alkhalifah, T., 2017. Selective data extension for full waveform inversion-an efficient solution for cycle skipping, Geophysics, 83(3), 157.

Xue, Z., Alger, N. \& Fomel, S., 2016. Full-waveform inversion using smoothing kernels, in Proceedings o the SEG Technical Program Expanded Abstracts 2016, pp. 1358-1363, Dallas, Texas, USA.

Yang, Y., Engquist, B., Sun, J. \& Hamfeldt, B.F., 2018. Application of optimal transport and the quadratic Wasserstein metric to full-waveform inversion, Geophysics, 83(1), R43-R62.

Yuan, Y.O. \& Simons, F.J., 2014. Multiscale adjoint waveform-difference tomography using wavelets, Geophysics, 79(3), WA79-WA95.

Zalesak, S.T., 1979. Fully multidimensional flux-corrected transport algorithms for fluids, $J$. Comput. Phys., 31(3), 335-362.

Zheng, H., Zhang, Z. \& Liu, E., 2006. Non-linear seismic wave propagation in anisotropic media using the flux-corrected transport technique, Geophys. J. Int., 165(3), 943-956. 